\title{
A Theoretical Assessment of the Effects of Vector-Virus Transmission Mechanism on Plant Virus Disease Epidemics
}

\author{
L. V. Madden, M. J. Jeger, and F. van den Bosch
}

First author: Department of Plant Pathology, Ohio State University, Wooster 44691-4096; second author: Department of Agriculture and Horticulture, Wye College, University of London, Wye, Ashford, Kent TN25 5AH, United Kingdom; and third author: Department of Statistics, IACR-Rothamsted Experimental Station, Harpenden, Hertfortshire AL5 2JQ, United Kingdom. Accepted for publication 31 January 2000.

ABSTRACT

\begin{abstract}
Madden, L. V., Jeger, M. J., and van den Bosch, F. 2000. A theoretical assessment of the effects of vector-virus transmission mechanism on plant virus disease epidemics. Phytopathology 90:576-594.

A continuous-time and deterministic model was used to characterize plant virus disease epidemics in relation to virus transmission mechanism and population dynamics of the insect vectors. The model can be written as a set of linked differential equations for healthy (virus-free), latently infected, infectious, and removed (postinfectious) plant categories, and virus-free, latent, and infective insects, with parameters based on the transmission classes, vector population dynamics, immigration/emigration rates, and virus-plant interactions. The rate of change in diseased plants is a function of the density of infective insects, the number of plants visited per time, and the probability of transmitting the virus per plant visit. The rate of change in infective insects is a function of the density of infectious plants, the number of plants visited per time by an insect, and the probability of acquiring the virus per plant visit. Numerical solutions of the differential equations were used to determine transitional and steady-state levels of disease incidence $\left(d^{*}\right) ; d^{*}$ was also determined directly from the model parameters. Clear differences were found in disease development among the four transmission classes: nonpersistently transmitted (stylet-
\end{abstract}

borne [NP]); semipersistently transmitted (foregut-borne [SP]); circulative, persistently transmitted $(\mathrm{CP})$; and propagative, persistently transmitted (PP), with the highest disease incidence $(d)$ for the SP and CP classes relative to the others, especially at low insect density when there was no insect migration or when the vector status of emigrating insects was the same as that of immigrating ones. The PP and CP viruses were most affected by changes in vector longevity, rates of acquisition, and inoculation of the virus by vectors, whereas the PP viruses were least affected by changes in insect mobility. When vector migration was explicitly considered, results depended on the fraction of infective insects in the immigration pool and the fraction of dying and emigrating vectors replaced by immigrants. The PP and CP viruses were most sensitive to changes in these factors. Based on model parameters, the basic reproductive number $\left(R_{0}\right)$-number of new infected plants resulting from an infected plant introduced into a susceptible plant population-was derived for some circumstances and used to determine the steady-state level of disease incidence and an approximate exponential rate of disease increase early in the epidemic. Results can be used to evaluate disease management strategies.

Additional keywords: compartmental model, nonlinear model, strategic modeling, theoretical epidemiology.
Viruses with insect and other arthropod vectors cause many economically important plant diseases of both annual and perennial crops in tropical and temperate regions $(37,39)$. Disease incidence depends on many factors including the number and behavior of vectors, the resistance of plants to the viruses and vectors, and the virus transmission process $(14,35,36)$. Transmission class is of fundamental importance for characterizing the relationship between a plant virus and its (primarily homopteran) insect vector (25) and is putatively a key factor in virus disease development under a given set of environmental and host conditions (2). However, little effort has been made in determining the influence of the insect-virus interaction on the resulting epidemics in the general sense (8). Understanding this relationship could lead to improved selection of management strategies for different virus diseases. We show in this paper that nonlinear modeling is a useful way to understand the linkages between transmission class and plant disease development.

We follow Nault (28) and use a classification system that combines the systems based on the persistence of the virus in the vector $(40,41)$ and the mode (or mechanism) of transmission (20). Persistence is defined in terms of the retention time of the plant virus in its vector, and three categories have been designated: nonpersistent, semipersistent, and persistent. Mode of transmission can

Corresponding author: L. V. Madden; E-mail address: madden.1@ osu.edu

Publication no. P-2000-0403-03R

(C) 2000 The American Phytopathological Society be categorized as stylet-borne, foregut-borne, circulative, and propagative. Combining these produces four classes: (i) nonpersistently transmitted, stylet-borne ('nonpersistent' [NP]); (ii) semipersistently transmitted, foregut-borne ('semipersistent' [SP]); (iii) circulative, persistently transmitted ('circulative-persistent' or simply circulative $[\mathrm{CP}]$ ); and (iv) propagative, persistently transmitted ('propagative-persistent' or simply propagative [PP]). For the propagative-persistent class, the virus multiplies in the insect vector as well as in the plant host. Nault (28) provided a thorough synthesis of the transmission processes and showed the value of these classes for systematics and ecology.

It is well known that certain management tactics work best only for viruses in some transmission classes $(34,37)$. For instance, insecticide sprays often are not effective for nonpersistent viruses, because the pesticide does not work fast enough to prevent virus acquisition or inoculation $(34,37)$. In fact, increased (but temporary) mobility of the vectors sprayed with insecticide may lead to increased disease incidence. On the other hand, the propagative-persistent viruses are more readily controlled with insecticides $(31,34)$. However, other than the insecticide tactic, there has been little research on the relationship between transmission class and disease control, in particular, or disease dynamics, in general. There are many ways of assessing the impact of virus transmission class on epidemics and effectiveness of controls, ranging from field experiments involving specific pathosystems to modeling of virus disease dynamics in relation to transmission properties. An advantage of the latter is that one can focus on consequences of differ- 
ent transmission classes for fixed conditions of vector dynamics (independent of the plant virus) and host susceptibility.

Before 1998, only Ferriss and Berger (8) had explicitly focused on the consequences of transmission class on resulting epidemics. They used a discrete-time, stochastic simulation approach to model the spread of hypothetical virus diseases in a spatial lattice of plants. Their excellent paper showed, among other things, that transmission class can have a large effect on disease development. Their model was somewhat limited by lack of reproduction in the plant and vector populations, fixed and equal number of vectors and virus transmission probabilities for all transmission classes, and relatively short time durations of the epidemics. Jeger et al. (18) recently proposed a more general, deterministic, continuous-time population-dynamic model for plant virus diseases in relationship to insect vector dynamics that is analogous to some models developed for human pathogens with vectors (such as malaria [32]), but explicitly considers the characteristics of the four transmission classes. Jeger et al. (18) determined equilibrium or steady-state values of plant disease and insect populations in order to determine conditions that resulted in persistence of disease in the plant population. Large differences in steady states were found between the propagative viruses and the others for the situation with no insect migration. Only limited numerical results were determined for epidemic behavior over the full range of time values during an epidemic, and numerical results and analyses of steady states were not presented for the case of explicit insect migration.

The objective of this paper is to expand on the theoretical work of Jeger et al. (18) to gain insight into how transmission class can affect epidemics caused by plant viruses with arthropod vectors. To accomplish this objective, we explore properties of the plant virus disease model under a range of conditions involving either no explicit vector migration or with vector immigration and emigration. We use the results to evaluate the implications of transmission class on some disease management strategies.

\section{THEORY AND APPROACHES}

Virus transmission. The four transmission classes can be distinguished by the (mean) times for virus acquisition, inoculation (transmission), and latent period in the insect vector (28). Consider the time line in Figure 1, in which $T$ represents the time that an insect is 'in contact' with a plant. $T$ can be thought of as feeding or probing time by an insect, but there is no assumption that the vector is actually feeding (probing) throughout this time. If a virus-free insect is placed on an infected and infectious (discussed below) plant at $T=0$, acquisition proceeds at a rate of $\lambda$ (units of per time), corresponding to a mean acquisition time in a population of insects (if no mortality occurs) of $1 / \lambda$ (units of time [e.g., days]). The virus passes through the latent period in the vector at a rate of $\eta$, corresponding to a mean latent period (in a population) of $1 / \eta$. For some classes, $1 / \eta$ is 0 . The insect vector then becomes infective (or inoculative) and passes through this infectious period at a rate of $\tau$, corresponding to a mean time of $1 / \tau$ for a population

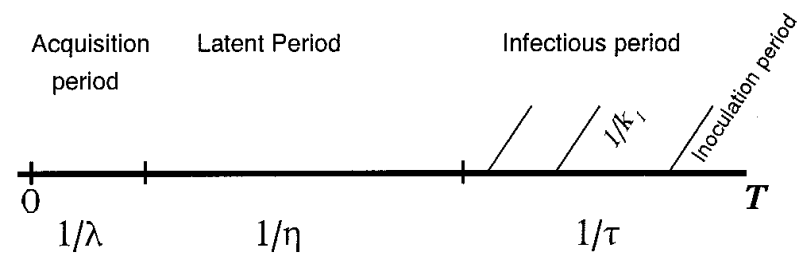

Fig. 1. Time line of an insect vector feeding on a plant, starting at time (T) 0 . Virus acquisition occurs at a rate of $\lambda$ from a virus-infected (and infectious) plant, the virus passes through the latent period in the vector at a rate of $\eta$, and the virus passes through the infectious period in the vector at a rate of $\tau$. When feeding on a virus-free (healthy) plant, inoculation occurs at a rate of $k_{1}$ (diagonal time lines show inoculations). of insects (when there is, again, no mortality). Infective indicates that the insect is capable of transmitting the virus to a healthy (susceptible and virus-free) plant. Values of $1 / \tau$ can be as large as the life of the insect to as small as an hour (or less). Once an insect is no longer infective, it can reacquire the virus from an infected plant. A vector is said to be viruliferous from the time it acquires a virus until the virus is lost. The sum of $1 / \eta$ and $1 / \tau$ is often termed the virus retention time.

If an infective insect feeds on a virus-free plant (Fig. 1, the diagonal time lines intersecting the infectious period time line), it transmits at a rate of $k_{1}$, corresponding to a mean time of inoculation of $1 / k_{1}$ (when there is no mortality). An individual infective insect can transmit a virus to more than one plant, given sufficient time and access.

Times for acquisition, inoculation, and length of retention in the vector are often determined for plant viruses $(2,28)$. Many studies, however, record the minimum times for a population of insects rather than the mean or median (Discussion in literature citation 2). Because the minimum (threshold time) is heavily influenced by the number of insects (and plants) tested, it is difficult to determine exact values for the parameters $1 / \lambda, 1 / \eta, 1 / \tau$, and $1 / k_{1}$. However, approximate order-of-magnitude values can be easily specified based on a general assessment of the published values. Nault (28) indicated that acquisition time is of the order of seconds to minutes, minutes to hours, hours to days, and hours to days for the nonpersistent, semipersistent, circulative, and propagative transmission classes, respectively. Latent periods are essentially 0 for nonand semipersistent viruses, and of the order of hours to days and weeks for the circulative and propagative viruses, respectively. Retention time (latent plus infectious period) is of the order of minutes, hours to days, days to weeks, and weeks to months for the four classes (28). These values are for normal conditions when the vectors are feeding and moving among plants in the same field or nearby fields. It is known that the retention time can be much longer for the nonpersistent class when vectors are not 'in contact' with plants, such as when they are traveling in jet streams over large distances (3).

Based on Nault (28) and Berger and Ferriss (2), we have chosen nominal values for these parameters (Table 1). We further assume here that times for acquisition and inoculation are the same within each class, but these times can be (very) different across classes. Values in Table 1 are given in units of days, with corresponding hours presented in parentheses for some very short times. Based on these parameter values, non- and semipersistent viruses are acquired and transmitted very quickly, with essentially no time lapse between acquisition and inoculation. Insects also quickly lose the ability to transmit the viruses in these two classes, such that reacquisition is needed for continued inoculations. Conversely, propagative and, to a lesser extent, circulative viruses are acquired (and transmitted) slowly, with a substantial period of time between acquisition and inoculation; however, the insect remains infective for a long time.

TABLE 1. Relevant parameters for the four plant virus transmission classes: nonpersistently transmitted, stylet-borne (NP); semipersistently transmitted, foregut-borne (SP); circulative, persistently transmitted (CP); and propagative, persistently transmitted (PP)

\begin{tabular}{lllll}
\hline $\begin{array}{l}\text { Time and other } \\
\text { parameters }^{\mathrm{a}}\end{array}$ & \multicolumn{1}{c}{$\mathrm{NP}$} & $\mathrm{SP}$ & \multicolumn{1}{c}{ CP } & PP \\
\hline Acquisition $(1 / \lambda)$ & $0.021(0.5 \mathrm{~h})$ & $0.083(2 \mathrm{~h})$ & $0.5(12 \mathrm{~h})$ & $2.0(48 \mathrm{~h})$ \\
Inoculation $\left(1 / k_{1}\right)$ & $0.021(0.5 \mathrm{~h})$ & $0.083(2 \mathrm{~h})$ & $0.5(12 \mathrm{~h})$ & $2.0(48 \mathrm{~h})$ \\
Latent period $(1 / \eta)$ & 0 & 0 & 1 & 20 \\
Infectious period $(1 / \tau)$ & $0.25(6 \mathrm{~h})$ & 4 & 20 & $\infty$ \\
Viruliferous progeny $(q)$ & 0 & 0 & 0 & 0.5 \\
\hline
\end{tabular}

${ }^{a}$ Parameters refer to the plant virus interaction with the insect vector. All listed parameters are times, except for $q$, which is unitless. Units shown are days (with hours in parentheses for some short times). 
A further characteristic that separates propagative from the other classes is transovarial transmission of the virus from females to progeny. For this class, a fraction of the eggs laid $(q)$ are infected by the plant virus. For the other transmission classes, $q$ is 0 .

Model. Plant population. The plant population consists of $K$ individuals that are partitioned into four nonoverlapping compartments (stages, categories, or states): healthy or susceptible (virus free) $(H)$, latently infected $(L)$, infectious $(S)$, and removed $(R) . H$, $L, S$, and $R$ represent the numbers of plants in each category (Fig. 2 ). The number of diseased individuals $(D)$ is given by $L+S+R$ or by $K-H$. When an individual plant becomes infected (discussed below), it moves through the latent stage at a rate of $k_{2}$ (units of per time [e.g., per day]) into the infectious stage, corresponding to a mean latent period in a population of $1 / k_{2}$ (days) when there is no mortality (Fig. 2). Latent indicates that the plant is infected but that insect vectors are not yet able to acquire the virus from the plant because sufficient virus multiplication and intraplant spread has not yet occurred. For annual crops, $1 / k_{2}$ is generally at least 5 days for most viruses; mean latent periods can be much longer for perennials. When a plant is infectious, a virusfree insect vector can acquire the virus, assuming that it feeds from an infectious plant for a sufficient period of time.

Infected plants lose infectiousness and proceed into the removed or postinfectious stage at a rate of $k_{3}$ (units of per time), giving a mean infectious period of $1 / k_{3}$ when there is no mortality (Fig. 2). The removed state occurs because the virus titer becomes too low for acquisition or the diseased plant no longer is attractive for insect feeding (Fig. 1 in literature citation 6). Values of $1 / k_{3}$ can be as long as the life of the plant or be relatively short. When a plant is removed, an insect can no longer acquire the virus from the infected plant. In the model, plants do not recover from infection; that is, removed plants do not become susceptible. The val- ues of $1 / \eta$ and $1 / \tau$ for the vector-virus interaction are not related to the values of $1 / k_{2}$ and $1 / k_{3}$ for the plant-virus interaction.

Plants die in the model in all categories at a rate of $\beta$ (per time), independent of infection status in the model. To maintain a constant plant population size, dead plants are replaced at the same rate, $\beta$ (Fig. 2). This would be analogous to the replanting of dead plants in an orchard or to the production of new plant units (e.g., tillering) as plants die. This rate can, of course, be 0 .

Plant infection and rate equations. Linked differential equations were specified directly for the four plant categories $(H, L, S$, and $R$ ) based on the specifications given here, rather than directly specifying an equation for $D$. This is the standard approach in medical epidemiology (1). An equation for $D$ can then be determined using algebra. The equation for $H$ can be written generally as

$$
\frac{d H}{d t}=f(H)-\Lambda H
$$

in which $f(H)$ is a function for change in host population size independent of disease, and $\Lambda$ is the so-called force of infection, the probability per unit of time that a healthy (susceptible) plant becomes infected (1). Based on the assumptions described in the previous paragraph, we set $f(H)$ equal to $\beta(K-H)$. To directly account for insect transmission, $\Lambda$ must be based on the number of infective insects $(Z)$ rather than on the number of infectious plants $(S)$ or number of diseased plants $(D)$, as is commonly done for simpler models $(4,6,23)$. We specify the following expression for $\Lambda$ as

$$
\Lambda=\phi b Z / K
$$

in which $\phi$ is a parameter for the number of plants visited per time period (e.g., per day) by an insect, and $b$ is the probability of inoculating the plant per visit (a value between 0 and 1). Determi-

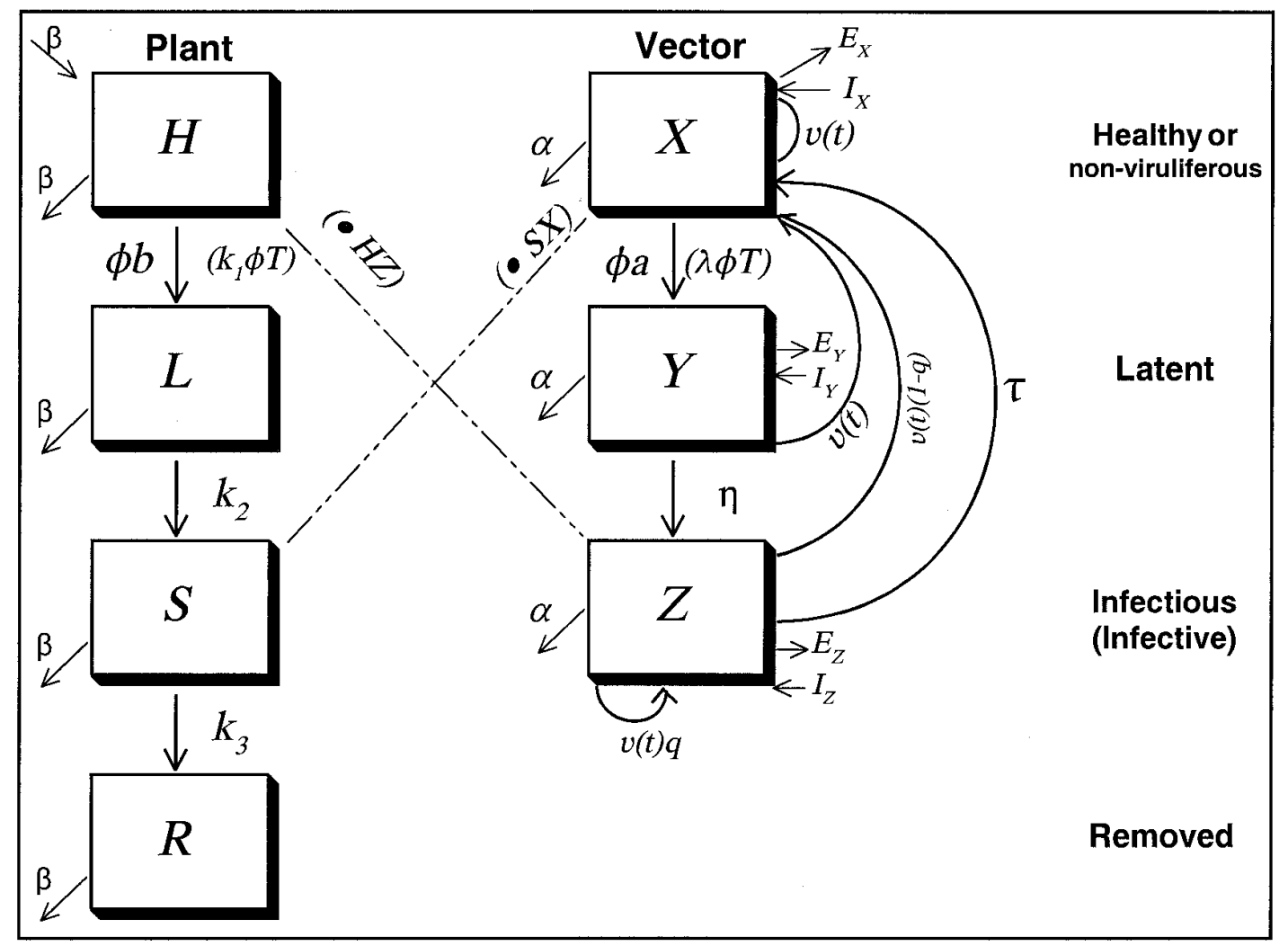

Fig. 2. Schematic of the plant-virus insect-vector model. Variables are in boxes, and parameters are listed next to the lines and arrows. Tables 1 and 2 have an explanation of terms. For ease of presentation, full specification of model terms are not given. For instance, the insect emigration rate parameters (e.g., $\left.E_{X}\right)$ are multiplied by the insect numbers in a given category $\left(E_{X} \cdot X\right)$, but the immigration rates (e.g., $\left.I_{X}[t]\right)$ are arbitrary functions of time. Equations 4 and 6 have details. Diagonal lines represent the 'contact' between infective insects and healthy plants, and between infectious plants and virus-free insects. 
nation of $Z$ is explained below. Plants become infected based on the 'contact' between healthy plants $(H)$ and infective insects $(Z)$, which is modeled as a product of these two terms and the rate constant $\phi b$ (Fig. 2).

The value of $b$ can be calculated based on mean inoculation times $\left(1 / k_{1}\right)$ (Fig. 1) as

$$
b=1-e^{-k_{1} T}
$$

in which $T$ is the time of feeding per plant visit. Equation 3 can be derived from the Poisson distribution. Using the values of the transmission class parameters (Table 1), predicted values of $b$ are shown in Figure 3. For small values of $k_{1}$ and $T$, equation 3 is approximately equal to $k_{1} T$, so that $\phi b$ in equation 2 is $k_{1} \phi T$. $\phi T$ can then be considered the fraction of each day that an insect feeds (or is 'in contact' with a plant). This was the formulation used by Jeger et al. (18). However, we use equation 3 directly in the expression for the force of infection (equation 2) to avoid obtaining unrealistically high values of the product $k_{1} \phi T$ for some transmission classes.

Using equation 2 for the force of infection, the linked differential equations for the plant population are

$$
\begin{aligned}
\frac{d H}{d t} & =\beta(K-H)-\phi b \frac{Z H}{K} \\
\frac{d L}{d t} & =\phi b \frac{Z H}{K}-\left(k_{2}+\beta\right) L \\
\frac{d S}{d t} & =k_{2} L-\left(k_{3}+\beta\right) S \\
\frac{d R}{d t} & =k_{3} S-\beta R
\end{aligned}
$$

The equation for $R$ is redundant when the plant population size is fixed $(R=K-[H+L+S])$, but is included here for completeness.

Equation 4 is the mathematical equivalent of the left-hand column of Figure 2. The last term of $d H / d t$ and first term of $d L / d t$ represent both the decline in the number of healthy plants and increase in the number of diseased plants (which start as latently infected individuals). The $k_{2} L$ term in $d L / d t$ and $d S / d t$ represents the transition from the latent to infectious state; the $k_{3} S$ term in $d S / d t$ and $d R / d t$ represents the transition from the infectious to removed state. The $\beta$ terms represent the plant mortality and replanting rates. Specifically, the products of $\beta$ with $L, S$, and $R$ represent mortality, whereas the product of $\beta$ and $H$ represents new growth.

Insect vector population. In addition to the infective state $(Z)$, insects can be classified with respect to plant viruses as being virus free $(X)$ and in the latent $(Y)$ state (Fig. 2), in which $X, Y$, and $Z$ represent the numbers in each category (Fig. 2). Total insect population size is $P(P=X+Y+Z)$. As described above for the trans-

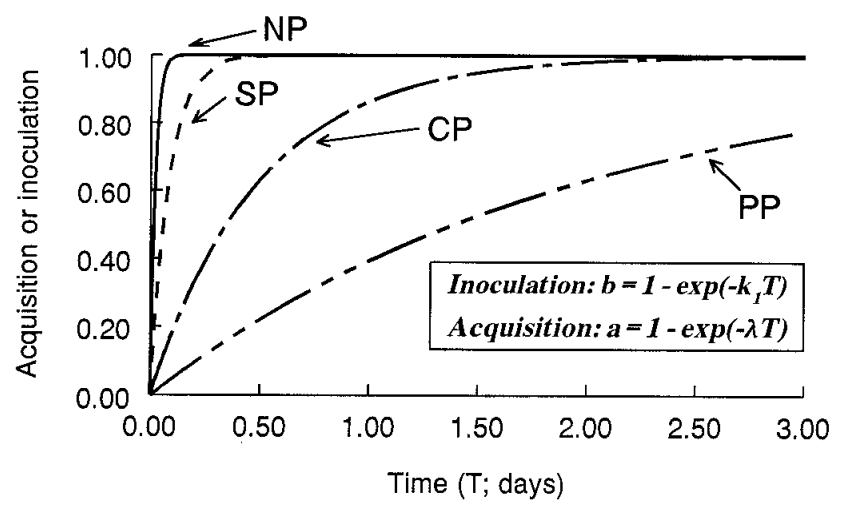

Fig. 3. Probability of an insect acquiring a virus from a plant $(a)$ or transmitting (inoculating) a virus to a plant $(b)$ in relation to time of feeding $(T)$ for nonpersistent (NP), semipersistent (SP), circulative-persistent (CP), and propagativepersistent (PP) classes, based on values of parameters in Table 1. mission classes (Fig. 1), when an insect acquires the plant virus, the virus passes through the latent state at a rate of $\eta$ and through the infectious state (back to the virus-free state) at a rate of $\tau$ (Table 1).

Population sizes $(X, Y$, and $Z)$ are affected by reproduction ('birth') and death. Except for propagative viruses (in which $q>0$ ),

TABLE 2. Variables and parameters in the plant virus-vector model

Term Explanation (initial conditions or nominal values)

$t \quad$ Time during an epidemic $(0 \rightarrow \infty$; nominal: $0 \rightarrow 200$ days $)$

$T \quad$ Time feeding (probing) per plant visit (nominal: $0.5 / \phi$ )

$K \quad$ Total plant population size (nominal: 1,000$)$

$L \quad$ Number of latently infected plants $\left(L_{0}=0.002 K\right) ; l=L / K$

$S \quad$ Number of infectious plants $\left(S_{0}=0\right) ; s=S / K$

$R \quad$ Number of removed (postinfectious) plants $\left(R_{0}=0\right) ; r=R / K$

$H \quad$ Number of healthy (susceptible and virus-free) plants $\left(H_{0}=K-\left[L_{0}+\right.\right.$ $\left.\left.S_{0}+R_{0}\right]\right) ; h=H / K$

$D \quad$ Diseased plants $\left(D_{0}=L_{0}+S_{0}+R_{0}\right) ; d=D / K=1-h=l+s+r$

$d^{*} \quad$ Equilibrium (steady-state) value of $d$ corresponding to a dynamic plant host $(\beta>0 /$ day) (equation 12 )

$d_{\infty} \quad$ Asymptotic value of $d$ as $t$ goes to $\infty$, when there is no steady state (because $\beta=0 /$ day) (equation 13)

$\Lambda \quad$ Force of infection (equation 2)

$P \quad$ Number of insect vectors (nominal: depends on transmission class) $P / K \quad$ Insects density (per plant)

$Y \quad$ Number of insects in latent state (stage or category) $\left(Y_{0}=0.005 P\right)$; $y=Y / K$

$Z \quad$ Number of infective insects $\left(Z_{0}=0\right) ; z=Z / K$

$Z / P \quad$ Fraction of infective insects in the vector population

$X \quad$ Number of virus-free insects $\left(X_{0}=P-\left[Y_{0}+Z_{0}\right]\right) ; x=X / K$

$1 / k_{1} \quad$ Time to inoculate a plant by an insect vector ${ }^{\mathrm{a}}$

$1 / k_{2} \quad$ Latent period of virus in plant (nominal: 5 days)

$1 / k_{3} \quad$ Infectious period of virus in plant (nominal: 12.5 days)

$\beta \quad$ Plant mortality and replanting (regrowth) rate (nominal: 0.01/day)

$\phi \quad$ Plants visited per day by an insect (nominal: $1 /$ day)

$1 / \lambda \quad$ Time to acquire a plant virus by a vector ${ }^{\mathrm{a}}$

$1 / \eta \quad$ Time for plant virus to move through latent state in the vector (latent period) ${ }^{\mathrm{a}}$

$1 / \tau \quad$ Time for plant virus to move through infectious state in the vector (infectious period) ${ }^{\mathrm{a}}$

a Probability of acquisition of a plant virus from an infectious plant by an insect per plant visit (equation 5)

$b \quad$ Probability of virus inoculation of a plant by an infective insect per plant visit (equation 3)

$\alpha \quad$ Insect population mortality rate in full (variable- $P$ ) model (equation 6); or turnover rate ('birth' and death rate) in fixed- $P$ Model I (equation 9) (nominal: 0.2/day)

$v(t) \quad$ Arbitrary total fecundity ('birth') rate of insect population (per day) for within-field reproduction; for fixed- $P$ Model I: $v(t)=\alpha \cdot(X+Y+Z)$

$E_{X} \quad$ Emigration rate for virus-free insects (nominal: 0/day) in the full (variable- $P$ ) model

$E_{Y} \quad$ Emigration rate for insects in latent state (nominal: 0/day)

$E_{Z} \quad$ Emigration rate for infective insects (nominal: 0/day)

$I_{X}(t) \quad$ Immigration rate for virus-free insects (nominal: 0/day)

$I_{Y}(t) \quad$ Immigration rate for insects in latent state (nominal: 0/day)

$I_{Z}(t) \quad$ Immigration rate for infective insects (nominal: 0/day)

$q \quad$ Probability of a vector offspring being viruliferous ${ }^{\mathrm{a}}$

$V \quad$ Vector emigration rate for Model II (equation 14), total (and fixed) number of vectors that emigrate per day (nominal: equivalent to 0/day for the no-explicit-migration Model I)

$\psi \quad$ Fraction of emigrating and dead vectors replaced by within-field reproduction in Model II (equation 14) (nominal: equivalent to 1 for Model I)

$\theta_{1} \quad$ Fraction of immigrants that are virus free in Model II (equation 14) (nominal: not relevant for Model I)

$\theta_{2} \quad$ Fraction of immigrants in the latent state in Model II (equation 14) (nominal: not relevant for Model I)

$1-\theta_{1}-\theta_{2}$ Fraction of immigrants that are infective in Model II (equation 14) (nominal: not relevant for Model I)

$\sigma \quad a \phi / \tau$ (derived parameter for the limit when $\eta=\tau=\infty$ ) (equation 18); applicable for nonpersistent viruses only

$R_{0} \quad$ Basic reproductive number (equation 11)

$r_{E} \quad$ Exponential rate of disease increase early in an epidemic; directly relevant only for Model I (equation 16)

$\mu \quad$ Mean age of a infectivity of an individual (plant or insect) (equation 17)

a Table 1 has nominal values for the four transmission classes. 
all progeny of insects are virus free in the model, meaning that only $X$ increases due to reproduction for the other three transmission classes. Total population fecundity (reproductive or 'birth') rate at time $t$ is given by the general expression $v(t)$. When $q>0$, both $X$ and $Z$ can increase due to reproduction, thus, the fecundity rate in the $X$ state is reduced from the total $v(t)$ to

$$
v(t)-v(t) q\left(\frac{Z}{X+Y+Z}\right)
$$

in which $Z /(X+Y+Z)=Z / P$ is the fraction of infective insects in the population. The rate of change of $Z$ increases by $v(t) q Z /(X+Y+Z)$ when $q>0$, because some offspring are infective. Probability of dying in a unit of time is $\alpha$, assumed to be the same for each state; $X, Y$, and $Z$ thus decrease at rates of $\alpha X, \alpha Y$, and $\alpha Z$, respectively.

Values of $X, Y$, and $Z$ also are affected by immigration and emigration (Fig. 2). We assume that immigration rates are independent of insect numbers within a field, but possibly vary with time. Thus, $X, Y$, and $Z$ in a given plant virus pathosystem can increase at rates of $I_{X}(t), I_{Y}(t)$, and $I_{Z}(t)$, respectively. On the other hand, emigration is assumed to depend on the numbers of insects within the field. Thus, $X, Y$, and $Z$ decrease at rates of $E_{X} X, E_{Y} Y$, and $E_{Z} Z$, respectively. (It should be noted here that the schematic in Figure 2 shows the variables and parameters of relevance, but it does not fully reflect every rate term considered. For instance, to avoid clutter in the figure, it is not specified which terms involve multiplication with a variable [e.g., $\left.E_{X} X\right]$ and which ones do not [e.g., $\left.I_{X}\right]$ ).

Insect vectors acquire the virus based on the 'contact' between virus-free insects $(X)$ and infectious plants $(S)$. The rate is given by $\phi a X S / K$, in which $\phi$ is as previously defined, and $a$ is the probability of a virus-free insect acquiring the virus per plant visit. As with $b$ in equation $4, a$ can be determined from

$$
a=1-e^{-\lambda T}
$$

in which $\lambda$ and $T$ are shown in Figure 1. Although Jeger et al. (18) used the approximation $\lambda \phi T$ for $\phi a$ (because $a \approx \lambda T$ when $\lambda$ and $T$ are small), we prefer to use $\phi a$ directly in the equations to ensure realistic 'contact' rate results for large $\lambda$. Although $b$ goes to 1 quickly with increasing $T$ (Fig. 3), especially for the nonpersistent and semipersistent viruses, this does not indicate that $100 \%$ of vectors are viruliferous at any single time. This is because of the short infectious periods for these classes $(1 / \tau)$, meaning that the virus is lost rather quickly by insects for these transmission classes.

Based on the model assumptions given here, the differential equations for $X, Y$, and $Z$ can be written as

$$
\begin{aligned}
\frac{d X}{d t} & =v(t)-v(t) q\left(\frac{Z}{X+Y+Z}\right)+\tau Z+I_{X}(t)-E_{X} X-\alpha X-\phi a \frac{S X}{K} \\
\frac{d Y}{d t} & =\phi a \frac{S X}{K}+I_{Y}(t)-E_{Y} Y-\alpha Y-\eta Y \\
\frac{d Z}{d t} & =\eta Y-\tau Z+I_{Z}(t)-E_{Z} Z+v(t) q\left(\frac{Z}{X+Y+Z}\right)-\alpha Z
\end{aligned}
$$

Simplifications and scaling. Equations 4 and 6 can be used to represent a plant virus epidemic under very general circumstances for insect population development. Although there is no analytical solution for linked differential equations of this type, numerical integration is straightforward. Because of the large number of terms for insect development (equation 6), including fecundity ('birth') and mortality (death) rates and immigration and emigration rates in each category, it is difficult to focus on the general effects of transmission class on the epidemics. Thus, some simplifications are justified to gain insight on transmission. The primary simplification used here is to specify a constant size of the insect population $(P=X+Y+Z)$. Essentially, this converts the variable $P$ into a parameter. The individual insect classes $(X, Y$, and $Z)$ all still vary over time, but the total is fixed.
For convenience of interpretation, the variables in equations 4 and 6 were scaled by dividing by $K$, the total number of plants. The new variables are $h=H / K, l=L / K, s=S / K, r=R / K, x=X / K, y=$ $Y / K$, and $z=Z / K$. With this scaling, $h+l+s+r=1$, and $d(=[L+$ $S+R] / K)$ is given by $1-h$ and $l+s+r$. Also, $x, y$, and $z$ represent the numbers of insects per plant (density terms) and $P / K=x+y+z$.

Two forms of the model can be considered as further simplifications. The first stipulates that loss of insects due to emigration are made up by immigration in the same category. For instance, if 10 vectors in the $Z$ category emigrate, 10 infective insects immigrate into the field. This is based on a supposition that the population of insects is the same globally as locally. From a mathematical perspective, this is equivalent to making all the immigration and emigration terms 0 . With a fixed $P$, 'births' and deaths are balanced, resulting in the following equality.

$$
v(t)=\alpha \cdot(X+Y+Z)
$$

$\alpha$ is then both a fecundity ('birth') and death rate (Table 2) and can be considered a turnover rate of the insect population. High values of $\alpha$ indicate a short life span of insects, and low values indicate that individuals live for long times; when $\alpha=0$ /day, there are no 'births' or deaths, and the same insects are present for the entire time period of the epidemic.

With the scaling of variables, the plant equations can be written after algebraic manipulation as

$$
\begin{aligned}
& \frac{d h}{d t}=\beta(1-h)-\phi b z h \\
& \frac{d l}{d t}=\phi b z h-\left(k_{2}+\beta\right) l \\
& \frac{d s}{d t}=k_{2} l-\left(k_{3}+\beta\right) s
\end{aligned}
$$

with the equation for $d r / d t$ omitted because of redundancy (because $r=1-[h+l+s])(18)$. The insect equations can be written as

$$
\begin{gathered}
\frac{d y}{d t}=\phi a s\left(\frac{P}{K}-y-z\right)-\alpha y-\eta y \\
\frac{d z}{d t}=\eta y-\tau z-\alpha z+q \alpha z
\end{gathered}
$$

with the equation for $d x / d t$ omitted because $x=P / K-y-z$. We call this Model I. For the nonpersistent and semipersistent classes, $y$ (or $Y$ ) equals 0 , indicating that the rate of transfer through the $Y$ category is infinite. One way of obtaining numerical solutions with equation 9 for the non- and semipersistent classes is to use a large value for $\eta$ as an approximation. An alternative, which was not presented by Jeger et al. (18), is to mathematically eliminate the equation for $d y / d t$ (because $\eta=\infty$ ), producing the single equation for the vectors

$$
\frac{d z}{d t}=\phi a s\left(\frac{P}{K}-z\right)-\tau z-\alpha z+q \alpha z
$$

Because $q=0$ for these classes, $q \alpha z$ can also be removed from equation 10 .

This continuous-time model (equations 8 and 9 [or 10]) is similar to several other compartmental models of plant and animal diseases $(1,6,10,15)$ (chapter 9 in literature citation 4$)$, except that the force of infection (equation 2) is based on $Z$ and not $S$ (the vector is explicitly considered). It is also similar to some vector-pathogen models $(12,13,23,24,27,32)$, but is more general because all four categories of disease in the host population are explicitly considered and proper choice of model parameters (Table 1) permits the representation of epidemics caused by all four transmission classes.

Equilibria. Jeger et al. (18) found that nonzero equilibria or steady states can exist for plant and insect categories under some circumstances (parameter values) if $\beta$ is greater than $0 /$ day. A nonzero steady state for $d\left(d^{*}\right)$ means that disease can persist in the plant population. It is possible to determine the conditions necessary for 
the disease to persist by equating the differential equations (equations 8 and 9 [or 10]) to 0 and by (extensive) algebraic manipulations to calculate the combinations of parameters that give a nonzero value of $z$ (and, hence, $s$ and $d$ ). Some of the equilibrium equations are given in the Appendix.

Based on the steady-state expressions, a threshold for persistence of disease can be determined in terms of model parameters and, from this threshold, the basic reproductive number $\left(R_{0}\right)$ can be derived (7) for the plant virus epidemic. For the simpler differential-delay model of Vanderplank (38), $R_{0}$ is known as the progeny-parent ratio (" $i R$ " using the symbols of Vanderplank, different from those used here). $R_{0}$ is the (average) number of new infected plants that result from one infected plant introduced into a susceptible population. When $R_{0}<1$, the disease will not persist; conversely, the disease persists if $R_{0}>1$ (7). Thus, this fundamental term is also critical in determining if 'invasion' of the disease can occur, that is, if an introduction of a diseased plant into a population results in an epidemic. If $R_{0}<1$, each diseased plant does not 'replace itself' with another diseased plant, and an epidemic does not occur. More precisely, when $R_{0}<1$, the fraction of infectious plants in the diseased population $(s /[l+s+r])$ decreases to 0 over time. If $R_{0}>1$, each diseased plant results in more than one new diseased plant (when the population of plants is susceptible).
The equation for $R_{0}$ based on equations 8 and 9 (or 10) is given by

$$
R_{0}=\left(\frac{P}{K}\right)\left(\frac{a b \phi^{2}}{\tau^{\prime} k_{3}^{\prime}}\right)\left(\eta^{\prime} k_{2}^{\prime}\right)
$$

in which $\tau^{\prime}=\tau+\alpha(1-q), k_{3}{ }^{\prime}=k_{3}+\beta, \eta^{\prime}=\eta /(\alpha+\eta)$, and $k_{2}{ }^{\prime}=$ $k_{2} /\left(k_{2}+\beta\right)$. Here, $\tau^{\prime}$ and $k_{3}{ }^{\prime}$ are the inverses of the mean times (in a population with mortality) that an infective insect stays in the infective state and the plant stays in the infectious state, respectively. $k_{2}{ }^{\prime}$ is the probability of an infected plant becoming infectious, which is virtually 1 for low natural mortality values (small $\beta$ ); $\eta^{\prime}$ is the probability of an insect that had acquired the virus becoming infective, which is 1 (or nearly so) for all transmission classes except, possibly, the propagative one at large $\alpha$. For nonpersistent viruses, $a b \phi^{2}$ in equation 11 is related to the concept of vector propensity proposed by Irwin and Ruesink (14).

Example model epidemics. Use of the model is demonstrated in Figure 4 for the propagative transmission class. Numerical solutions here (and later), unless explicitly specified otherwise, are based on the parameter values in Table 1 for the corresponding transmission class and on the nominal values in Table 2 for other parameters and initial conditions. Mathcad version 8 (MathSoft, Inc., Cambridge, MA) was used for all numerical integrations. The Bulirsch-Stoer method was utilized to numerically solve the differential equations. The left column of subfigures shows the results
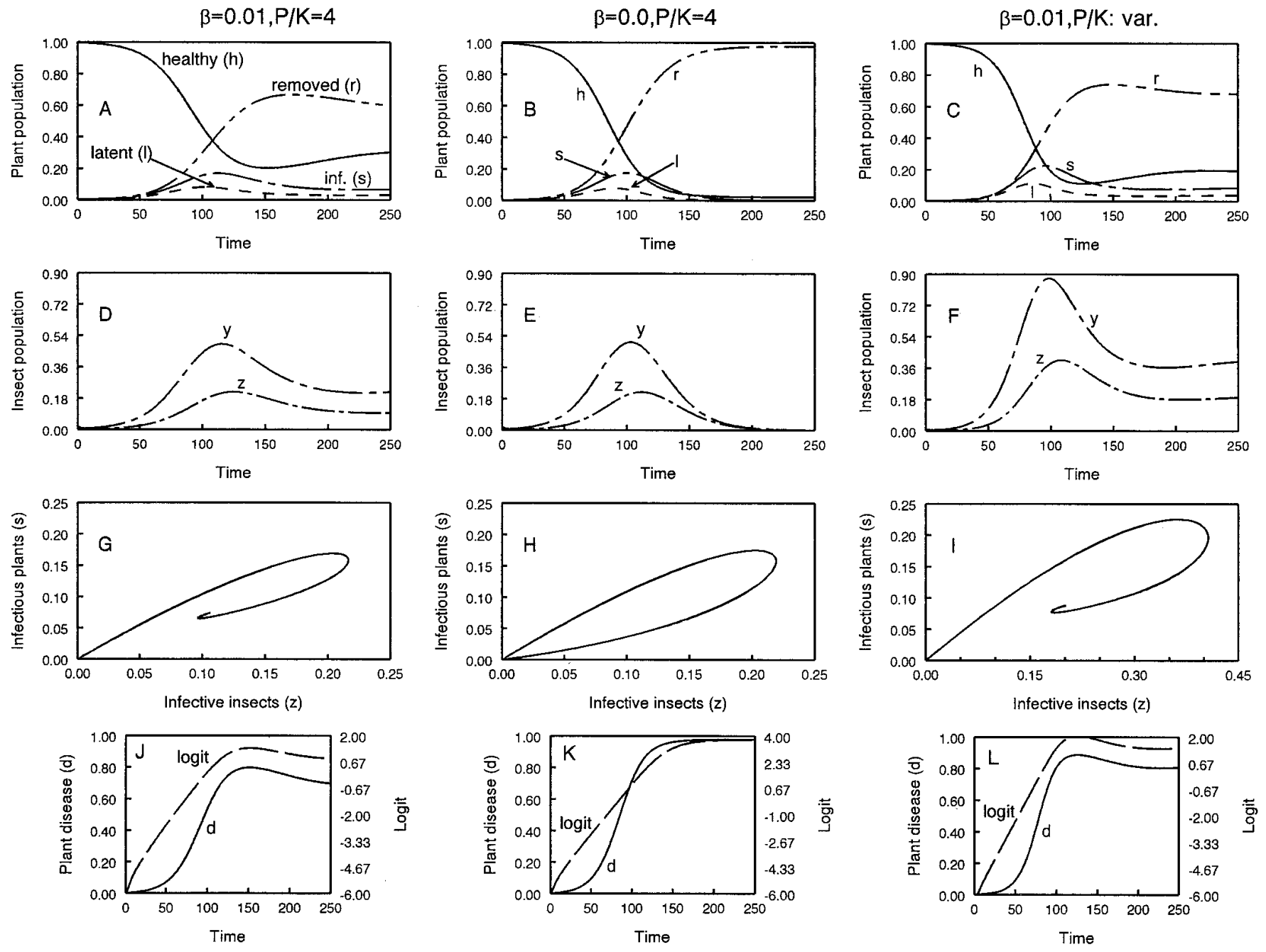

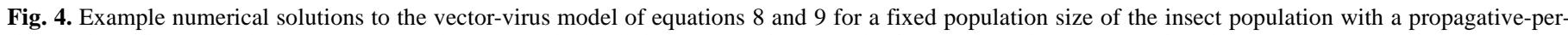

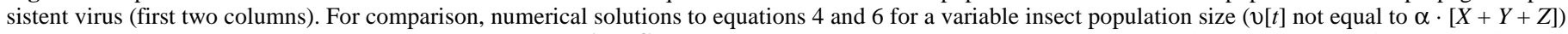

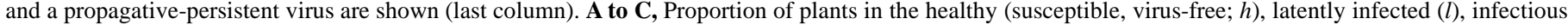

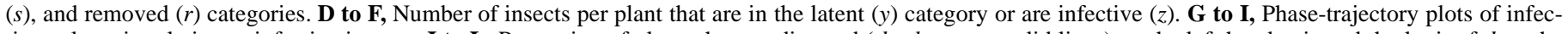

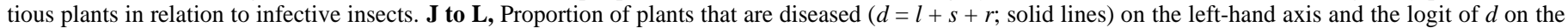
right-hand axis (long dashed lines). 
based on equations 8 and 9 for fixed $P / K$ and a plant mortality (and replanting) rate of $\beta=0.01 /$ day. The parameter conditions correspond to an $R_{0}$ of 3.8 (equation 11); that is, each diseased plant produces, on average, 3.8 new diseased plants in a population of susceptible individuals. The healthy plant population declines, initially, in a negative exponential or reverse-sigmoidal fashion as the other disease categories increase (Fig. 4A). Eventually, a minimum value of $h$ is reached and a very slight increase then occurs because of the continuing introduction of new healthy plants (because $\beta>0 /$ day). Both latent and infectious plant disease ( $l$ and $s$, respectively) increase to maxima around day 100 to 110 and then decline. Removed disease $(r)$ increases to a maximum of around 0.7 . Because $R_{0}>1$, all four plant variables in Figure 4A approach nonzero equilibria at large times, meaning that the disease persists in this system (Appendix) (18).

Total disease incidence $(d=1-h=l+s+r)$ increases in this epidemic to around day 150 and then declines somewhat (Fig. 4J). The value of $d$ fluctuates around an equilibrium $\left(d^{*}\right)$ of 0.71 at large times (equations A3 and A4). We have found that a general analytical expression for the disease equilibrium value in terms of $R_{0}$ can be given as

$$
d^{*} \leq 1-\left(R_{0}\right)^{-1}
$$

The equality is approached as $\beta$ goes to 0 (e.g., $\beta \lessgtr 0.001 /$ day). However, even with $\beta=0.01 /$ day, $1-\left(R_{0}\right)^{-1}$ gives a predicted $d^{*}$ of 0.73 with this example (Fig. $4 \mathrm{~J}$ ), relatively close to the exact model value of 0.71 obtained from equation A4. The logit of $d$ $(\operatorname{logit}[d])$ is also shown in Figure $4 \mathrm{~J}$; this variable increases in a mostly linear manner with $t$ up to day 150, indicating that disease progress was initially well described as an exponential and then a logistic process; closer to the $d^{*}$, the logistic nature of the epidemic clearly breaks down.

As the number of healthy plants declines (or $d$ increases), both the number of latent $(y)$ and infective $(z)$ insects per plant increase until around day 125 and then decline (Fig. 4D). Because of fixed $P$, virus-free insects are determined from $P / K-y-z$. Both $y$ and $z$ approached nonzero equilibria (equation A1 for $z^{*}$ ). In this example, the maxima and equilibria for $y$ and $z$ are considerably less than the fixed insect density of four insects per plant $(P / K)$. The change in healthy plants and virus-free insects is driven by infectious plants $(s)$ and infective insects $(z)$, as indicated by the first lines of equations 8 and 9; thus, it is informative to consider the phase plot of $s$ and $z$ (Fig. 4G), as done commonly in population dynamic modeling (26). In this example epidemic, $s$ and $z$ increase concurrently until $z$ is about 0.22 , both then decline until $z$ is about 0.1 and then there is an increase again. A simulation with much longer $t$ indicates that this trajectory quickly approaches the equilibrium values for these two variables (L. V. Madden, unpublished data).

The central column of Figure 4 corresponds to equations 8 and 9 , with the same parameters and initial conditions as the left-hand column, but with $\beta=0 /$ day, indicating that plants do not die (or are replanted) during the epidemic. For this epidemic, $R_{0}=4.4$ (equation 11). For the first 90 days of the epidemic, the change in the four plant categories (Fig. 4B), as well as the insect categories (Fig. 4E), was nearly the same as when $\beta=0.01 /$ day (Fig. 4A and D). A plot of $d$ versus $t$ was also sigmoidal.

Because of the lack of replanting, there are no nonzero equilibria, and $h$ continues to decrease (and $d$ increase) at large times as $s$ and $z$ go to 0 (Fig. $4 \mathrm{H}$ and $\mathrm{K}$ ). However, $h$ does not reach 0 , or, equivalently, $d$ does not reach 1 at very large times. This is because the fraction of diseased plants that are removed or postinfectious $(r / d)$ goes to 1 before $h$ reaches 0 ; in other words, there can be no further increase in $d$ if all diseased plants are postinfectious (15). In general, we have found that the asymptotic value of $d\left(d_{\infty}\right)$ lies between $1-\left(R_{0}\right)^{-1}$ and 1 . For a wide range of population-dynamic compartmental models (19) and for differential-delay models (16) with no mortality or regrowth of the host, the asymptotic result for $d$ is of the general form

$$
d_{\infty} \approx 1-\left(1-d_{0}\right) e^{-R_{0} d_{\infty}}
$$

The asymptotic value of $d$ for the example in the center column of Figure 4 is 0.978 (Fig. 4K), which agrees fairly well with the approximation of 0.988 obtained with equation 13 . However, we have not yet determined the exact expression for the relation between $d_{\infty}$ and $R_{0}$ when $\beta=0 /$ day in the model.

The third column of Figure 4 corresponds to the more general model that includes population growth of the vectors and explicit immigration and emigration (equations 4 and 6). Here, parameters are $K=1,000, P_{0}=2,000$ (giving an initial condition of $P_{0} / K=2$ insects per plant), $\alpha=0.1 /$ day, $I_{X}(t)=250$ insects per day (or 0.25 per plant per day), $I_{Y}(t)=I_{Z}(t)=0, E_{X}=E_{Y}=E_{Z}=0.04 /$ day, and $\beta=$ $0.01 /$ day. The vector 'birth' term was specified as

$$
v(t)=0.18(X+Y+Z)\left(1-\frac{X+Y+Z}{10,000}\right)
$$

which is a logistic function with a maximum population size of 10,000 insects (or 10 per plant) and a fecundity rate of $0.18 /$ day. Note that this fecundity rate is coupled with a mortality rate $(\alpha)$ of $0.1 /$ day and some nonzero immigration and emigration rates in the example. Other parameters are as listed in Tables 1 and 2. Numerical results were divided by $K($ e.g., $H / K=h)$ to give the same scale as used in equations 8 and 9.

This third example was chosen to show the robustness of the model by demonstrating that the epidemic outcome can be very similar for a fixed insect population size (left-hand column) and a variable population size. The behavior of the plant and insect categories over time are very similar between the left- and right-hand columns of Figure 4 (Fig. 4A and C, and Fig. 4D and F), and the phase plots of $s$ and $z$ show the same pattern (Fig. 4G and I). Alteration of the parameters listed in the previous paragraph could make the similarity even greater. Thus, the use of a fixed population size for the insect population was not found to be overly restrictive in interpreting epidemic results. The remainder of this paper deals with fixed $P$ as specified by equations 8 to 10 .

Model with explicit terms for vector immigration and emigration. The simplified plant virus epidemic model of equations 8 and 9 can be expanded for vector immigration/emigration, while maintaining a fixed $P$. One approach is to specify some additional parameters (18). Let $V$ represent the number of vectors that emigrate per time, which can take any value from 0 to $\infty$ per day. With fixed $P$, the emigrating and dead vectors must be replaced by either reproduction within the field ('births') or immigration. Thus, one can equate the following two expressions.

$$
v(t)+\left[I_{X}(t)+I_{Y}(t)+I_{Z}(t)\right]=V+\alpha \cdot(X+Y+Z)
$$

We define $\theta_{1}$ as the fraction of virus-free insects ( $X$ category) in the pool of immigrants and $\theta_{2}$ as the fraction of insects in the latent $(L)$ category in the pool of immigrants. We then let $1-\theta_{1}-\theta_{2}$ be the fraction of the immigrant pool that is infective ( $Z$ category). Furthermore, we let $\psi$ be the fraction of dying and emigrating (lost) insects replaced by reproduction inside the field. This means that $1-\psi$ is the fraction of dying and emigrating vectors replaced by immigration.

For this model, equation 8 for the plant population is unchanged and equation 9 is replaced, after extensive algebraic manipulation (18), by

$$
\begin{aligned}
& \frac{d y}{d t}=\phi a s\left(\frac{P}{K}-y-z\right)-\left(\alpha+\eta+\frac{V}{P}\right) y+(1-\psi) \theta_{2}\left(\frac{V+\alpha P}{K}\right) \\
& \frac{d z}{d t}=\eta y-\left[\tau+\left(\alpha+\frac{V}{P}\right)(1-q \psi)\right] z+(1-\psi)\left(1-\theta_{1}-\theta_{2}\right)\left(\frac{V+\alpha P}{K}\right)
\end{aligned}
$$


in which all terms are as defined previously (Table 2). We call this Model II. As with Model I, elimination of one of the vector equations is possible for the nonpersistent and semipersistent classes, because there are no insects in the latent category $(1 / \eta=0$ days $)$. The equation for infective insects becomes

$$
\frac{d z}{d t}=\phi a s\left(\frac{P}{K}-z\right)-\left(\tau+\alpha-q \alpha \psi+\frac{V}{P}-\frac{V}{P} q \psi\right) z+(1-\psi)\left(1-\theta_{1}\right)\left(\frac{V+\alpha P}{K}\right)
$$

For these two classes, $\theta_{2}=0$, by definition. Because $q=0$ for the nonpersistent and semipersistent classes, multiplication terms involving $q$ can also be removed from equation 15 .

Using these new parameters and equations 8 and 14 (or 15), a wide range of epidemic conditions can be specified. For instance, the simplified model without explicit emigration/immigration (Model I) is obtained when $V=0 /$ day (no emigration) and $\psi=1$ (all dying insects replaced by within-field reproduction). When $V=0$ and $\psi<$ 1 , there is no emigration, but some of the dead insects are replaced by immigrants. The extreme case of no colonization of the plant crop by vectors can be specified with $V=P$ and $\psi=0$ (all vectors emigrate per time period, and no 'births' within the field). Here, all emigrants are replaced by immigrants, and the $\theta$ terms specify the immigrants that are in the $X, Y$, and $Z$ categories. This case can be important for nonpersistent viruses (14).

Equations for equilibrium or steady states based on equations 8 and 14 (or 15) can be obtained, but are very complicated (Appendix) (18). If $\psi<1$-so that some of the insects lost to death and emigration (if $V>0$ ) are replaced by immigrants - and either $\theta_{2}$ or $1-\theta_{1}-\theta_{2}$ is greater than 0 , then there is no threshold for disease to persist and, thus, no definable $R_{0}$. This is because there will always be an influx of some new viruliferous insects into the system, and infections of plants will result not just from infected plants in the field (through the activity of local vectors), but also from viruliferous individuals originating outside the field (through the activity of immigrant vectors). Thus, early in the epidemic (at small $t$ ), disease increase is a mixture of exponential and linear processes. However, when $\theta_{1}=1$ (meaning that all immigrants are virus free), there is a threshold for persistence, and $R_{0}$ is defined as in equation 11 , except that $\tau^{\prime}$ and $\eta^{\prime}$ are given by the more complicated expressions

$$
\tau^{\prime}=\tau+\left(\alpha+\frac{V}{P}\right)(1-q \psi)
$$

and

$$
\eta^{\prime}=\frac{\eta}{\alpha+\eta+\frac{V}{P}}
$$

Numerical solutions to equations 8 and 14 (or 15) over a wide range of time values have not been previously determined; the relationship between model parameters and $d^{*}$ has also not been ascertained.

Numerical methods. Model I. The effects of selected insect and virus parameters on plant virus epidemics were determined for each of the transmission classes for Model I (no explicit vector migration). Unless stated otherwise, the values of the transmission parameters given in Table 1 were used for the corresponding transmission class, together with the nominal values of the other parameters and the initial level of the variables (e.g., $L_{0}$ for initial latently infected plants) given in Table 2.

Initially, the vector parameters that are unrelated to plant viruses (at least in the model) were evaluated, namely, $P / K, \alpha$, and $\phi$ (Table 2). These terms reflect vector density, mortality rate (and, hence, mean vector lifetimes because of the fixed $P$ ), and mobility, respectively. The following values of $P / K$ were tested: 0.05 , $0.1,0.2,0.4,0.8,1.6,3.2$, and 6.4 vectors per plant. Three values of $\alpha$ were evaluated, $0,0.2$, and $0.4 /$ day, and five values of $\phi$ were tested, $0.5,0.75,1,2$, and 3 plants per day. For the evaluations of $\alpha$ and $\phi$, the nominal values of $P / K$ were selected for the four transmission classes based on the results from the initial evalua- tion of $P / K$. That is, values of $P / K$ were chosen so that $R_{0}$ was the same $(=3.8)$ for the four transmission classes at the nominal values of $\alpha$ and $\phi$.

The virus-related parameters $k_{1}, \lambda, k_{2}$, and $k_{3}$ (Table 2) were then evaluated for their influences on epidemics. These terms reflect the rates at which inoculation and acquisition occur and the rates at which latently infected plants become infectious and then become postinfectious. Because both $k_{1}$ and $\lambda$ are based on the inherent properties of viruses in each transmission class, they cannot be varied as widely as some other terms without 'giving' a virus in one class the properties of another class. Thus, these terms were varied by $\pm 50 \%$ of the nominal values in Table 1 . For the cases shown here, $k_{2}$ and $k_{3}$ were also varied by $\pm 50 \%$. The other virus parameters of $\eta$ and $\tau$ were not tested, since there is less opportunity to alter these properties through management unless one could replace a wild-type virus with a less aggressive isolate in nature.

For selected numerical solutions of equations 8 and 9 (or 10) with the parameter values described above, the exponential rate of increase in $d\left(r_{E}\right)$ during the early part of the epidemics (when $d \lesssim 0.05$ ) was calculated. A plot of $\ln (d)$ versus $t$ was constructed for each model epidemic, which was found to be a straight line for small values of $t$ (L. V. Madden, unpublished data). An estimate of $r_{E}$ was obtained as the estimated slope of $\ln (d)$ versus $t$ for this linear portion of the graph, using ordinary least-squares regression.

For some population-dynamic processes, it is known that there is an approximate relationship between $r_{E}$ and $R_{0}$ (at small $R_{0}$ [5] and $d$ ) of the form

$$
r_{E} \cdot \mu \approx \ln \left(R_{0}\right)
$$

in which $\mu$ is the average age of infectivity of an individual (infected plant or viruliferous insect). For the numerical results, it was determined if equation 16 could describe the plant virus epidemic data when $\mu$ (5) was given by

$$
\mu=\frac{1}{2}\left[\left(\frac{1}{k_{2}+\beta}\right)+\left(\frac{1}{k_{3}+\beta}\right)+\left(\frac{1}{\eta+\alpha}\right)+\left(\frac{1}{\tau+\alpha}\right)\right]
$$

The first two terms within the brackets of equation 17 represent the mean for the plant population, and the last two terms represent the mean for the insect population.

Model II. The model with fixed $P$ (or $P / K$ ) and explicit vector immigration/emigration (Model II: equations 8 and 14 [or 15]) was used to determine the effects of vector migration on plant virus epidemics for the four transmission classes. The nominal values of the parameters from the nonmigration model (Model I: equations 8 and 9) were used, together with the value of $P / K$ that produced an $R_{0}$ of 3.8 with Model I. Selected values of vector emigration rate $(V / P)$, fraction of lost vectors (by death and emigration) replaced within the field by reproduction $(\psi)$, and fraction of infective $\left(1-\theta_{1}-\theta_{2}\right)$ immigrants were then tested for their effects on epidemics. In all cases, $\theta_{2}=0$ (no immigrant vectors in the latent state) was used in the model, for simplification of presentation.

In the first evaluation, $V / P$ values of 0.5 and 1.0 /day were considered at two extreme values of $1-\theta_{1}-\theta_{2}$ (Table 3$)$. When $V / P=$ $1 /$ day, all vectors in the system leave the system daily. For $V / P=$ $1 /$ day, we considered that all emigrants and insect deaths were replaced by immigrants (no 'births'; $\psi=0$ or $1-\psi=1$ ); for $V / P=$ $0.5 /$ day, we specified that half of the emigrants and deaths were replaced by immigrants and the rest replaced by within-field reproduction $(\psi=0.5)$. For each of the two $V / P$ values tested, we considered that all immigrants were either infective $\left(1-\theta_{1}-\theta_{2}=\right.$ $1)$ or virus free $\left(\theta_{1}=1\right.$, or $\left.1-\theta_{1}-\theta_{2}=0\right)$.

In the second evaluation of epidemics for Model II, we fixed $\theta_{1}$ at $0.9\left(1-\theta_{1}-\theta_{2}=0.1\right)$. Thus, $10 \%$ of the immigrating insects were infective in all cases, rather than the extreme cases of 0 and $100 \%$ in the evaluation in the previous paragraph. We considered the effects of emigration rate, $V / P$, ranging from 0 to 1 per day (Table 3), with all emigrating and dying insects replaced by immi- 
grants $(\psi=0)$. An additional treatment consisted of an intermediate $V / P$ and with all lost insects replaced by 'births' instead of immigration $(\psi=1)$.

\section{RESULTS}

Vector density. The propagative transmission class differed from the others in terms of the effects of vector density $(P / K)$ on plant virus epidemics (Fig. 5). In general, the semipersistent and circulative viruses had the highest disease incidence (and highest $R_{0}$ ) at any given value of $P / K$, followed by the nonpersistent and then by the propagative viruses. Although the nonpersistent class is characterized by the highest rates of virus inoculation $\left(k_{1}\right)$ and acquisition $(\lambda)$ (Table 1) (25), the inoculation $(b)$ and acquisition $(a)$ probabilities per plant visit are $\sim 1$ for this as well as the semipersistent class (equations 3 and 5) (Fig. 3). Thus, the very short infectious period $(1 / \tau)$ for the nonpersistent class results in lower disease levels than those for the semipersistent class. Likewise, although $a$ and $b$ are lower for the circulative compared with the nonpersistent class, the much longer infectious period for the former results in close to the same disease values as the semipersistent class. On the other hand, the even longer infectious period for the propagative class does not compensate for the low $a$ and $b$ values, and this class has the lowest levels of disease incidence at the tested $P / K$ values.

Numerical results for steady-state disease incidence $\left(d^{*}\right)$ agreed with the predicted values from equation A4 and were well approximated by $1-\left(R_{0}\right)^{-1}$ (equation 12 ). Disease incidence exceeded 0.5 with a $P / K$ of only 0.1 for the semipersistent virus class (at nominal values of the other parameters); to exceed this incidence value with a propagative virus, a $P / K$ between 1.6 and 3.2 was required (Fig. 5). Values of $P / K$ that resulted in an $R_{0}$ (equation 11) of 1 -the threshold for disease persistence-were $0.31,0.044$, 0.072 , and 1.07 vectors per plant for nonpersistent, semipersistent, circulative, and propagative classes (at the nominal values of the other parameters [Tables 1 and 2]), respectively. Model epidemics were very similar in shape to numerous published disease progress curves for perennial and annual crops $(4,22,30,35-37)$.
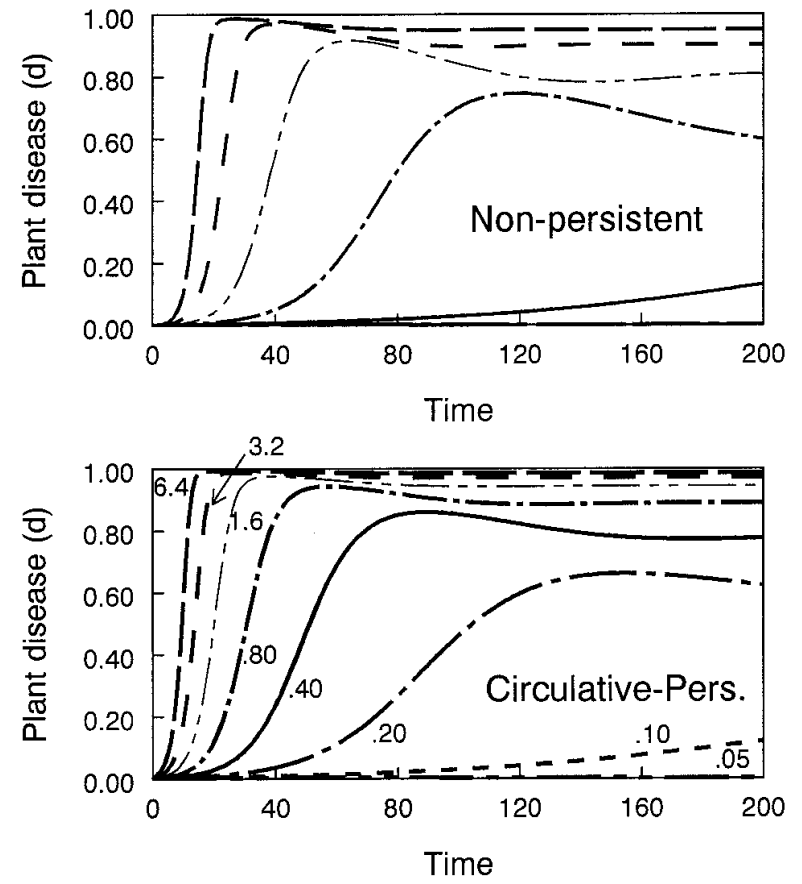

The effects of $P / K$ can also be seen in Figure 6A, in which disease incidence $(d)$ versus time $(t)$ is shown for epidemics with an $R_{0}$ of 3.8. Values of $P / K$ ranging from 0.2 to 4 vectors per plant were required to achieve this $R_{0}$. At large times, values of $d$ for all transmission classes approached a steady state of $\sim 0.71$ because of the equal $R_{0}$ values. However, Figure 6A also shows that the epidemic behavior in the short term (low to intermediate $t$ ) - the transient behavior-was not necessarily the same for the different classes. For instance, at $t \approx 75$ days, the nonpersistent virus class had the highest $d$, the propagative virus class had the lowest, and the other two virus classes were intermediate in terms of $d$. This

TABLE 3. Tested values of vector emigration rate $(V / P)$, fraction of virus-free $\left(\theta_{1}\right)$ and infective $\left(1-\theta_{1}-\theta_{2}\right)$ insects in the immigrant pool, and fraction of lost vectors (from mortality and emigration) replaced by within-field reproduction $(\psi)$ for the explicit vector migration model (Model II; equations 8 and $14[$ or 15$])^{\mathrm{a}}$

\begin{tabular}{|c|c|c|c|c|}
\hline Case label $^{\mathrm{b}}$ & $V / P$ & $\theta_{1}$ & $1-\theta_{1}-\theta_{2}$ & $\psi$ \\
\hline \multicolumn{5}{|l|}{ Evaluation 1} \\
\hline $\mathrm{i}$ & 0 & + & + & 1 \\
\hline ii & 1 & 0 & 1 & 0 \\
\hline iii & 1 & 1 & 0 & 0 \\
\hline iv & 0.5 & 0 & 1 & 0.5 \\
\hline $\mathrm{v}$ & 0.5 & 1 & 0 & 0.5 \\
\hline \multicolumn{5}{|l|}{ Evaluation 2} \\
\hline $0 / 1$ & 0 & + & + & 1 \\
\hline 0 & 0 & 0.9 & 0.1 & 0 \\
\hline 0.125 & 0.125 & 0.9 & 0.1 & 0 \\
\hline 0.25 & 0.25 & 0.9 & 0.1 & 0 \\
\hline $0.25 / 1$ & 0.25 & 0.9 & 0.1 & 1 \\
\hline 0.5 & 0.50 & 0.9 & 0.1 & 0 \\
\hline 1 & 1.0 & 0.9 & 0.1 & 0 \\
\hline
\end{tabular}

a Table 2 and text has a fuller description and units of parameters, and Table 1 has nominal parameter values with the four transmission classes. In all cases, the fraction of immigrants in the latent state $\left(\theta_{2}\right)$ was 0 .

${ }^{b}$ Disease progress curves are shown in Figures 12 and 13. Case $i$ and $0 / 1$ are equivalent to the model without explicit migration (Model I; equations 8 and 9 [or 10]).
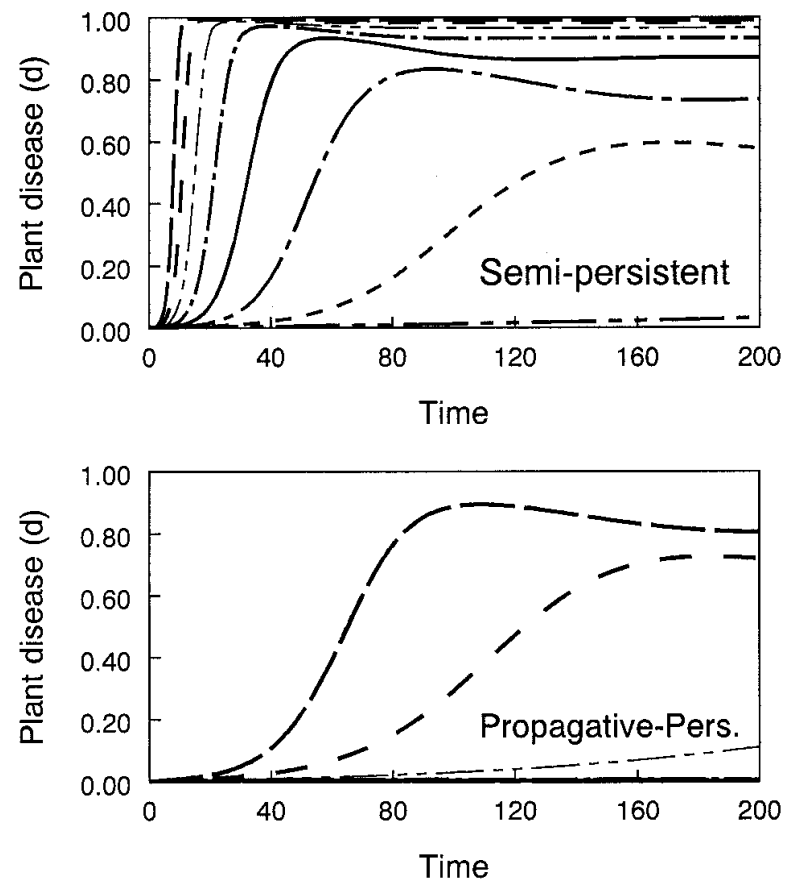

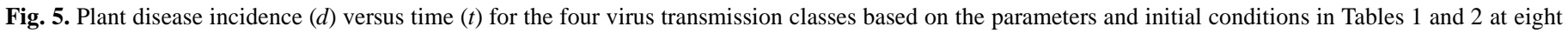

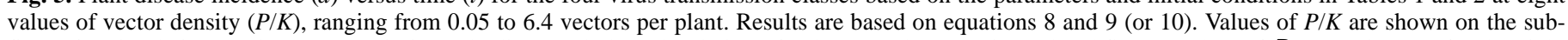

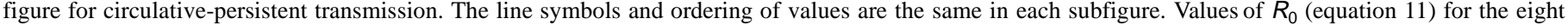

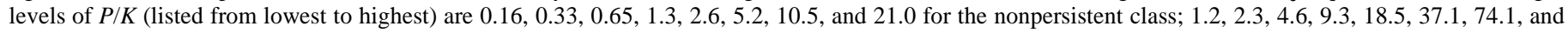

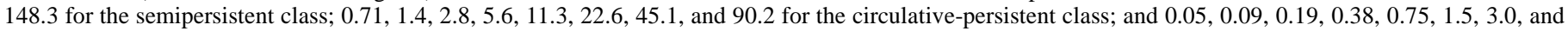
6.0 for the propagative-persistent class. 
was primarily due to the differences in the length of the latent $(1 / \eta)$ and infectious $(1 / \tau)$ periods in the vector (Table 1$)$ and the resulting $\mu$ (equation 17) among the four classes.

The change in infectious plant disease $(s)$ and infective vectors relative to the total vector population size $(Z / P=z /[P / K]=$ $[Z / K] /[P / K]$ ) for the four disease progress curves in Figure $6 \mathrm{~A}$ are shown in Figure 6B and C. The steady-state values of $s$ were about 0.07 (7\% of the plants) for all classes, but values were different among the four classes early in the epidemics. The time of maximum $s$ generally was associated with the length of the latent period in the vector $(1 / \eta)$, but not in a linear manner. The nonpersistent virus class had the highest value of $s$ at the time of the maximum, and the propagative class had the lowest (Fig. 6B). The other classes were intermediate.

Steady-state and maximum values of the fraction of vectors that are infective $(Z / P)$ varied with the transmission class, even at the same $R_{0}$ (Fig. 6C). There were similar steady states for the nonpersistent and propagative classes, which were considerably below the values for the other two classes. The maximum $Z / P$ was reached later for the propagative than for the nonpersistent class, due to the long latent period in the vector for the former class. The change in $Z / P$ with time for the semipersistent and circulative transmission classes was similar, except that the maximum was higher for the semipersistent viruses.

Vector mortality. The values of insect density $(P / K)$ that resulted in $R_{0}=3.8$ with nominal values of the other terms (Fig. 6) were used for the evaluation of insect mortality or turnover rate $(\alpha)$ and mobility $(\phi)$ on plant virus epidemics. Vector mortality/ turnover had a strong differential effect on epidemics for the four transmission classes (Fig. 7). For instance, there was virtually no effect of $\alpha$ on epidemics caused by nonpersistent viruses and a very large effect of $\alpha$ on epidemics for the circulative and propagative classes. The nonpersistent virus result is due to the short times needed for an insect to acquire and transmit the virus $(1 / \lambda$ and $1 / k_{1}$ ) relative to the life span of the insects, as well as the lack of latent period $(1 / \eta=0)$. That is, even if the individual vectors were only present for a very short time (high $\alpha$ ), there would still be sufficient time for acquisition and transmission. There was virtually no change in $R_{0}$ with change in $\alpha$ for this class (Fig. 7).

The long times needed for an insect to acquire and transmit a circulative or propagative virus and the long latent period in the vector, especially for the latter class, are the reasons why these transmission classes were sensitive to changes in $\alpha$. $R_{0}$ varied greatly with change in $\alpha$ for these classes (Fig. 7). With a high $\alpha$, there is insufficient time for acquisition and transmission; however, with small $\alpha$ (long mean life span), the long acquisition and inoculation times no longer were constraints. Note that $\alpha=0$ /day corresponds to the vectors living for the entire period of the epidemic, with no reproduction or deaths (analogous to the model in literature citation 8). With this minimum value of $\alpha$, disease incidence reached higher levels with the propagative transmission class than with the other classes in Figure 7. However, vector density $(P / K)$ was different for the four classes in this comparison. At $\alpha=0 /$ day and the same $P / K$ for all classes (e.g., $P / K=0.3$ ), disease incidence and $R_{0}$ for the propagative class were lower than for the circulative class, but still higher than for the nonpersistent class (L. V. Madden, unpublished data).

Vector mobility. The number of plants visited per day $(\phi)$ also had a large effect on epidemics (Fig. 8), but the sensitivity of the four classes to changes in this parameter was different compared with insect mortality/turnover (Fig. 7). In general, the nonpersistent and semipersistent classes responded most to changes in $\phi$, followed by the circulative and then the propagative classes (Fig. 8 ). Because increasing the number of plant visits per day necessitates that less time is available per visit to feed (and potentially acquire or transmit the virus), $T$ decreases with increasing $\phi$ (Table 2 ). With the very short acquisition and transmission times for nonpersistent and semipersistent viruses, a short feeding time per visit is not a constraint ( $a$ and $b$ [equations 3 and 5] are about 1 at all the $\phi[$ and $T]$ tested). Thus, increasing $\phi$ had a pronounced effect on epidemics for these transmission classes.

With viruses requiring long periods for acquisition and inoculation and a long latent period in the vector, the positive effects (in terms of higher $d$ ) of increasing $\phi$ could be partially negated by the decreased time $(T)$ available for these processes at each plant visit. For instance, with a propagative virus, a $\phi$ of 3 gives more opportunities for a viruliferous insect to transmit a virus to healthy plants, but each opportunity has a lower probability of success, because $b$ decreases as $\phi$ increases. For the propagative class, in particular, $a$ and $b$ both decrease substantially as $\phi$ increases. This resulted in a virtually unchanged $R_{0}$ with the parameters being used here (Fig. $8)$. However, there were some differences in $d$ at intermediate times (90 to 120 days).

Virus-plant interactions. The rate at which the virus passes through the latent $\left(k_{2}\right)$ and infectious periods $\left(k_{3}\right)$ in the plant (Fig. 2)
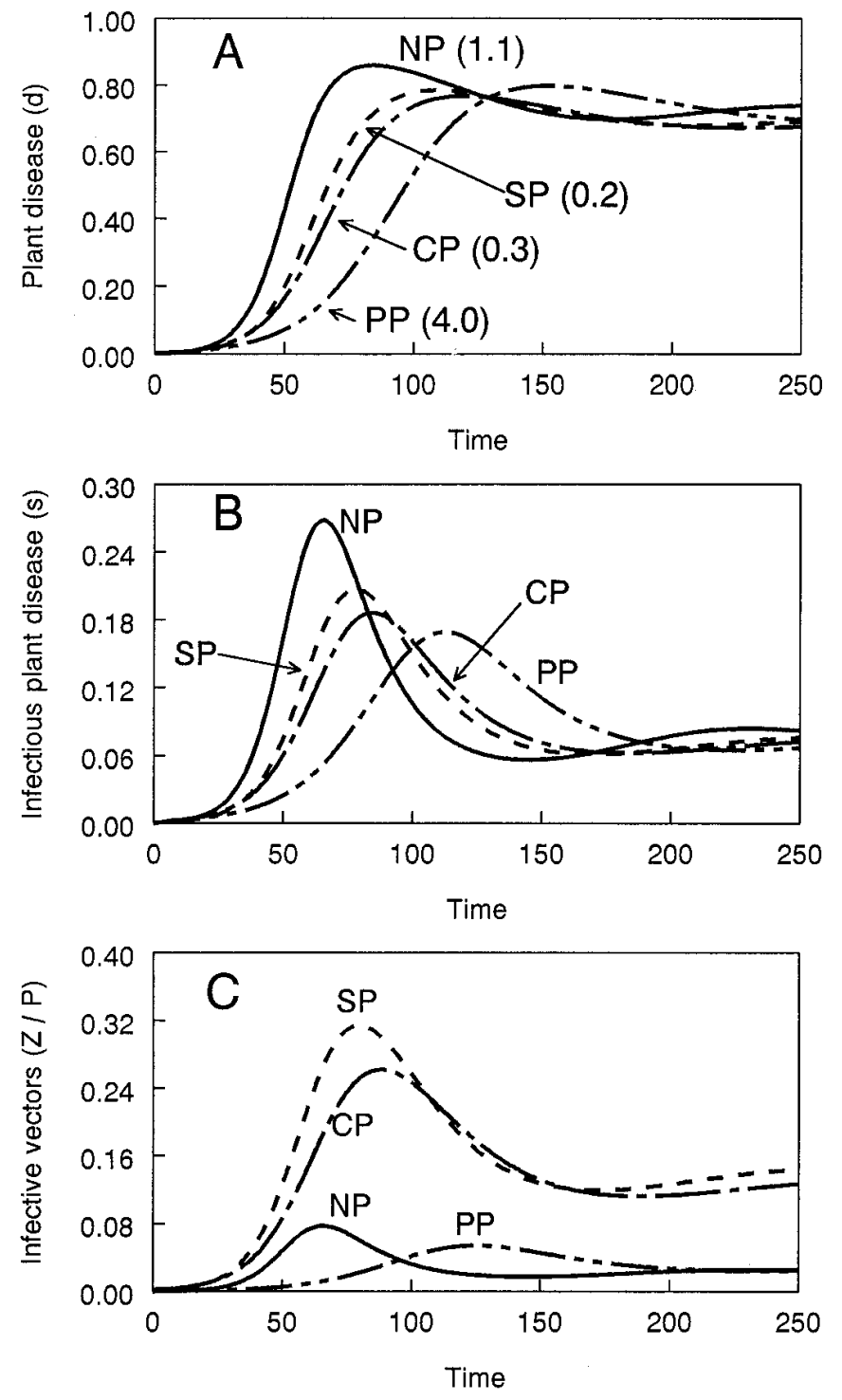

Fig. 6. A, Plant disease incidence $(d)$ versus time $(t)$ for the four transmission classes based on parameters and initial conditions in Tables 1 and 2, with levels of vector density $(P / K)$ selected to produce an $R_{0}$ (equation 11$)$ of 3.8 for each epidemic. Labels give the transmission class (Table 1) and the value of $P / K$ is in parentheses. B, Infectious plant disease $(s)$ versus time for the disease progress curves in $\mathbf{A}$ for the four transmission classes. C, Fraction of the vectors that are infective $(Z / P)$ for the disease progress curves in $\mathbf{A}$ for the four transmission classes. $\mu$ of equation 17 equals 8.1, 9.1, 10.4, and 12.4 days for the nonpersistent (NP), semipersistent (SP), circulative-persistent (CP), and propagative-persistent (PP) classes, respectively. 

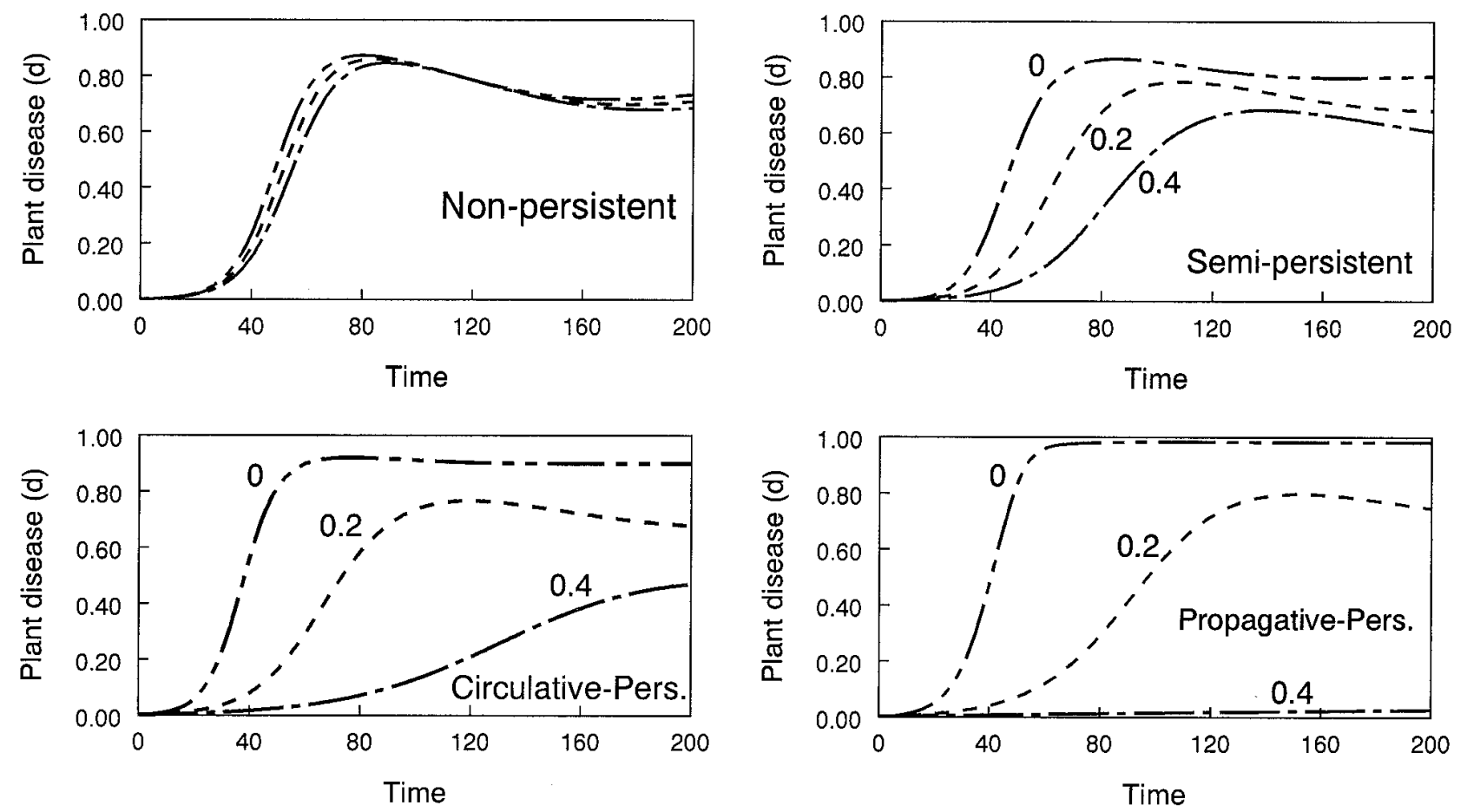

Fig. 7. Plant disease incidence $(d)$ versus time $(t)$ for the four virus transmission classes based on the parameters and initial conditions in Tables 1 and 2 at vector mortality/turnover rates $(\alpha)$ of $0,0.2$, and $0.4 /$ day. Vector density $(P / K)$ is $1.1,0.2,0.3$, and 4.0 for the nonpersistent, semipersistent, circulative-persistent, and propagative-persistent classes, respectively. Results are based on equations 8 and 9 (or 10). Values of $\alpha$ are shown next to the curves for some of the classes. Values of $R_{0}$ (equation 11) for the three $\alpha$ values (listed from lowest to highest $\alpha$ ) are 4.1, 3.8, and 3.5 for the nonpersistent class; 6.9, 3.8, and 2.6 for the semipersistent class; $22.8,3.8$, and 1.8 for the circulative-persistent class; and 207.1, 3.8, and 1.1 for the propagative-persistent class.
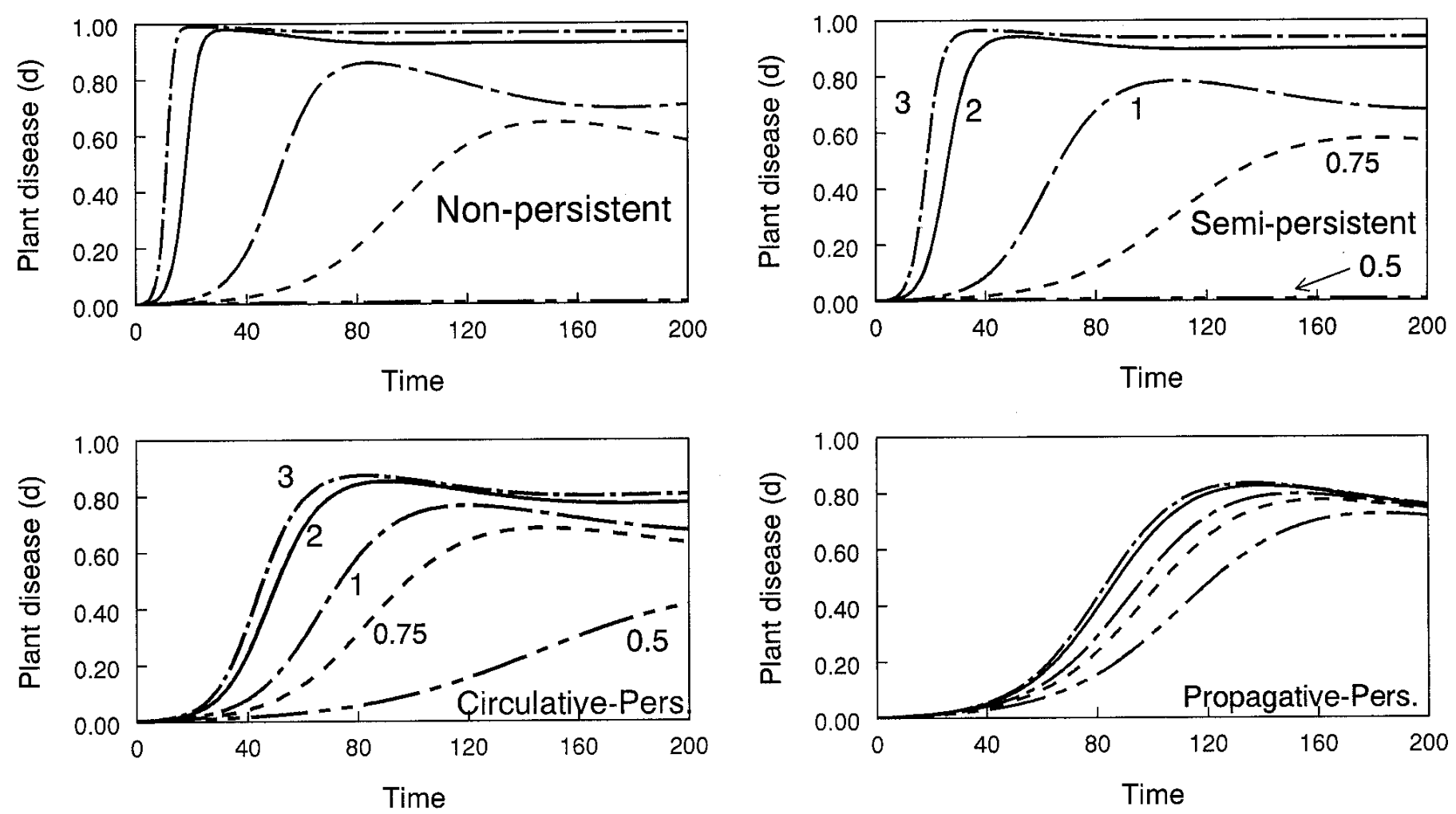

Fig. 8. Plant disease incidence $(d)$ versus time $(t)$ for the four virus transmission classes based on the parameters and initial conditions in Tables 1 and 2 at vector mobility rates $(\phi)$ of $0.5,0.75,1,2$, and 3 plant visits per day. Vector density $(P / K)$ is $1.1,0.2,0.3$, and 4.0 for the nonpersistent, semipersistent, circulative-persistent, and propagative-persistent classes, respectively. Results are based on equations 8 and 9 (or 10). Values of $\phi$ are shown next to the curves for some of the classes. Values of $R_{0}$ (equation 11) for the five $\phi$ values (listed from lowest to highest $\phi$ ) are $0.94,2.1,3.8,15.1$, and 33.9 for the nonpersistent class; $0.95,2.1,3.8,13.8$, and 25.7 for the semipersistent class; 1.8, 2.9, 3.8, 5.9, and 6.9 for the circulative-persistent class; and 2.9, 3.8, 3.5, 4.4, and 4.2 for the propagative-persistent class. 
had about the same effect on epidemics for all four transmission classes (L. V. Madden, unpublished data). This is demonstrated in Figure 9A and B for the nonpersistent class. There was no longterm (steady state) effect of $k_{2}$ on disease incidence, as expected from the small influence that $k_{2}$ has on $R_{0}$ (equation 11). However, the latent period did have a transitional effect, as seen around $t=$ 50 to 80 days in Figure 9A: increasing the length of the latent period (decreasing $k_{2}$ ) resulted in lower disease incidence during this time period.

The infectious period in the plant had both long-term (steady state) and short-term (transitional) effects on epidemics (Fig. 9B). The steady-state results were expected based on the large effect of $k_{3}$ on $R_{0}$ (equation 11). Increasing the length of the infectious period (decreasing $k_{3}$ ) resulted in a higher $R_{0}$ and disease incidence.

There was a differential response of the four transmission classes to change in the rate at which the virus is acquired $(\lambda)$ and inoculated $\left(k_{1}\right)$ or, equivalently, the mean time to acquire $(1 / \lambda)$ and transmit the virus $\left(1 / k_{1}\right)$. This is demonstrated for the nonpersistent and circulative-persistent classes in Figure 9C and D. For the transmission classes with short times to acquire and inoculate (nonpersistent [Fig. 9C] and semipersistent [data not shown]), change in these parameters had no effect on disease progress. This is because a $\pm 50 \%$ change in $\lambda$ and $k_{1}$ still resulted in values of $a$ and $b$ (equations 3 and 5) of around 1 per plant visit. However, for the transmission classes requiring long times for acquisition and inoculation (the two persistent classes), a change in $\lambda$ and $k_{1}$ had a large effect on $a$ and $b$ and the resulting epidemics. Increasing either or both of these rate terms (decreasing the mean times to acquire or transmit) resulted in a higher $R_{0}$ and disease incidence (Fig. 9D).

The effects of a simultaneous change $( \pm 50 \%$ of the nominal values) in $k_{1}, \lambda, k_{2}$, and $k_{3}$ on virus disease epidemics can be seen in Figure 10 . Here, a $50 \%$ increase in $k_{1}, \lambda$, and $k_{2}$ was coupled with a $50 \%$ decrease in $k_{3}$ (labeled $\times 1.5$, and termed an increase in the rates); conversely, a $50 \%$ decrease in $k_{1}, \lambda$, and $k_{2}$ was coupled with a $50 \%$ increase in $k_{3}$ (labeled $\times 0.5$, and termed a decrease in the rates). The two persistent classes were most affected by the simultaneous change in these four parameters, with no disease increase when the terms were reduced by $50 \%$ (giving an $R_{0}$ of less than 1) (Fig. 10). This can be attributed to the large effect that the rate of inoculation/acquisition and also the length of the infectious period have on disease progress for persistent viruses (Fig. 9D). However, changing the four parameters had only minor effects on the nonpersistent and semipersistent classes (Fig. 10). For these two classes, results were primarily due to the influence of $k_{3}$, since the other parameters had little individual effects on epidemics (Fig. 9A and B).

Exponential rate of disease increase. As exemplified in Figure $4 \mathrm{~J}$ to $\mathrm{L}$, there was a straight line between $\operatorname{logit}(d)$ and $t$ in most cases when $t$ was not large. This indicated that disease increase was approximately exponential at small $d(4,5)$. There was, in fact, a linear increase in $\ln (d)$ with $t$ at small times for the Model I epidemics summarized in Figures 5 to 10 when $R_{0}>1$ (L. V. Madden, unpublished data). Estimates of the exponential rate parameter, $r_{E}$, ranged from 0.01 to 0.78 /day for these cases. Using equation 16 and $\mu$ defined by equation $17, r_{E}$ could be well predicted for values of $R_{0}$ less than 15 (Fig. 11), although there was a slight overprediction by this equation.

Explicit vector migration. First evaluation. Vector immigration and emigration had a pronounced effect on the epidemics (Model II: equations 8 and 14 [or 15]), but the effects varied with transmission class (Fig. 12). In the first evaluation shown here, a situation with high vector emigration $(V / P=0.5$ or $1 /$ day) (Table 3$)$, the fraction of infective insects in the immigration pool $\left(1-\theta_{1}-\theta_{2}\right)$ determined the epidemic outcome. For the circulative-persistent and propagative-persistent transmission classes, there was no disease increase $\left(R_{0}<1\right)$ when all immigrants were virus free $\left(1-\theta_{1}-\right.$ $\theta_{2}=0$ and $\theta_{2}=0$ ) (Fig. 12, cases iii and v). This is because there was insufficient time for the immigrating insects to acquire or trans- mit the virus before emigrating. However, when $1-\theta_{1}-\theta_{2}$ equaled 0 for the nonpersistent class, there was virtually no effect on the epidemics relative to the nominal situation of no vector migration (Model I; case i). Because of the very high rates of acquisition and inoculation, coupled with the lack of latent period in the vector $(1 / \eta=0)$, there was sufficient time for the entire transmission process with nonpersistent viruses, even when there was no vector
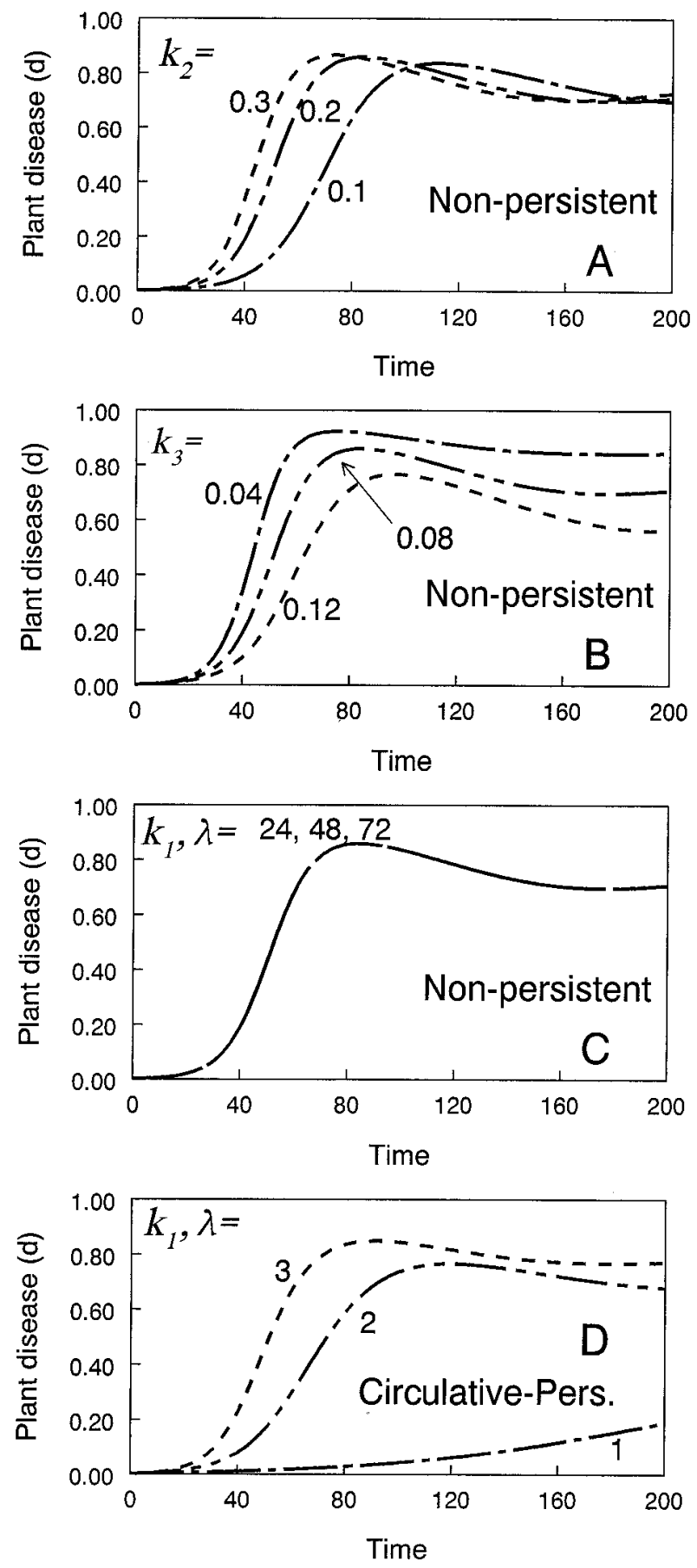

Fig. 9. Plant disease incidence $(d)$ versus time $(t)$ for the nonpersistent and circulative-persistent virus transmission classes based on the parameters and initial conditions in Tables 1 and 2 with changes in $\mathbf{A}, k_{2}$ (inverse of mean latent period in plant) for nonpersistent viruses; $\mathbf{B}, k_{3}$ (inverse of mean infectious period in plant) for nonpersistent viruses; $\mathbf{C}, k_{1}$ and $\lambda$ (rates of virus inoculation and acquisition) for nonpersistent viruses; and $\mathbf{D}, k_{1}$ and $\lambda$ for circulative-persistent viruses. Values of the parameters are indicated on the graphs. Values of $R_{0}$ (equation 11) for the three cases in each subfigure (listed from bottom to top curves) are $\mathbf{A}, 3.8,3.8$, and 3.8; $\mathbf{B}, 2.6,3.8$, and 6.8; $\mathbf{C}, 3.6$, 3.8, and 3.8; and D, 1.5, 3.8, and 5.7. Vector density $(P / K)$ is 1.1 and 0.3 for the nonpersistent and circulative-persistent classes, respectively. 
colonization (when all vectors emigrated daily). The semipersistent class was intermediate between the nonpersistent and the two persistent classes in terms of the effects of virus-free immigrants on disease progress. That is, disease incidence increased over time $\left(R_{0}>1\right)$ when $1-\theta_{1}-\theta_{2}$ equaled 0 , but incidence was less than the nominal situation with no vector migration.

Plant disease incidence approached 1 in the extreme situation of all immigrants being infective $\left(1-\theta_{1}-\theta_{2}=1\right)$, whether all $(V / P=$ $1 /$ day $)$ or half $(V / P=0.5 /$ day $)$ of the vectors emigrated per day (Fig. 12, cases ii and iv; Table 3 ). In these cases, $R_{0}$ is not defined, because $\theta_{1}<1$ (18). Transmission class had very little effect on the results. This combination of parameter values essentially created a simple-interest or monocyclic disease situation (4), especially for the two persistent classes. This is because an increase in $d$ was due to outside inoculum (infective immigrating vectors) rather than to spread from plant to plant, since there was no time for the latter. For this simple-interest process, increase in disease is linear at small $t$.

Second evaluation. Figure 13 shows the results of the second epidemic evaluation with Model II, in which the effects of a wide range of VIP values on disease progress were determined when $10 \%$ of the immigrants were infective $\left(1-\theta_{1}-\theta_{2}=0.1\right)$, rather than with the extremes of 0 and $100 \%$ in Figure 12. There was a much greater rate of disease development when lost insects (from death and emigration) were replaced by immigrants than by 'births.' This can be seen by comparing two pairs of epidemics in Figure 13. The first pair is the standard no-explicit-migration case (Model I: equivalent to $V / P=0 /$ day and $\psi=1$ [case label $0 / 1$ in Table 3]), and the case for $V / P=0 /$ day and $\psi=0$ (no emigration, but deaths replaced solely by immigrants [case 0]). The other pair is the case for $V / P=0.25 /$ day and $\psi=1$ (one-quarter of vectors emigrate daily, all replaced by within-field reproduction, and, thus, no introduction of virus into the field [case 0.25/1]) and the case for $V / P=0.25 /$ day and $\psi=0$ (emigrants and deaths replaced solely by immigrants [case 0.25 ]). The propagative-persistent class and, to a lesser extent, the circulative-persistent class were especially sensitive to within-field reproduction versus immigration (Fig. 13). This is because the fraction of infective insects in the total population (the variable $Z / P$ ) reached a higher steady state $(Z * / P)$ (equation A5) when $10 \%$ of the new vectors (the immigrants) were already infective (e.g., case 0.25 ) compared with the situation in which new vectors (from 'births') acquired the virus and became infective very slowly (e.g., case $0.25 / 1$ ). For example, $Z * / P$ for the circulative class equaled 0.14 for case 0.25 in Figure 13, but equaled only 0.03 for case $0.25 / 1$.

Epidemic behavior in relation to $V / P$ varied with transmission class when there was no reproduction in the field and immigrants replaced lost insects exclusively ( $\psi=0$ [cases 0 to 1 in Table 3]). Increasing $V / P$ led to lower disease development for the semipersistent and circulative classes (Fig. 13). This is because $Z * / P$ (equation A5) without emigration was higher than the value of $1-\theta_{1}-\theta_{2}$ used here (0.10). For instance, $Z * / P$ equaled 0.21 for the circulative transmission class at $V / P=0 /$ day (case 0 ), which was roughly double the value of $1-\theta_{1}-\theta_{2}$. Thus, increasing emigration rate $(V / P)$ led to replacing more and more infective insects with virusfree ones, diluting the infective individuals in the population, resulting in a lower $Z / P$ and, hence, $d$.

For the nonpersistent transmission class, the opposite result was seen; that is, increasing $V / P$ resulted in increasing the rate of disease development (Fig. 13). However, the reason is the same as for the semipersistent and circulative classes: $Z^{*} / P$ without emigration, but immigrants solely replacing dead insects, was low $\left(Z^{*} / P \approx\right.$ 0.03 [case 0]) and less than the $1-\theta_{1}-\theta_{2}$ parameter value used here. Thus, as emigration rate $(V / P)$ increased, the fraction of all insects that were infective increased (from the local acquisition of the virus and the increasing pool of infective immigrants), leading to higher $d$. For instance, $Z * / P \approx 0.04$ (equation A5) for $V / P=$ 0.25 /day with the nonpersistent class (case 0.25 ). Interestingly, for
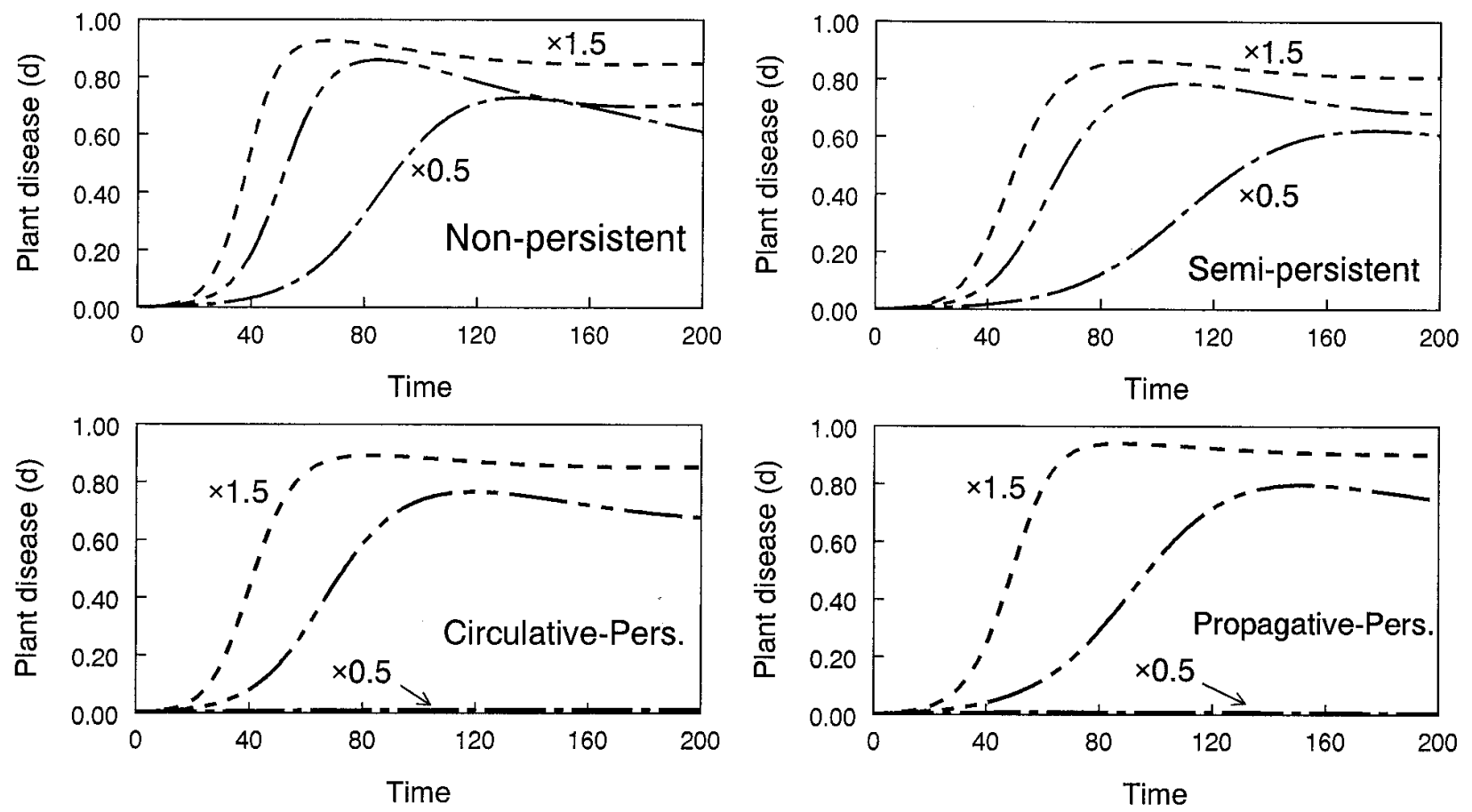

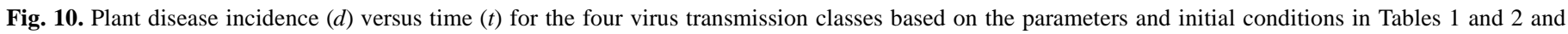

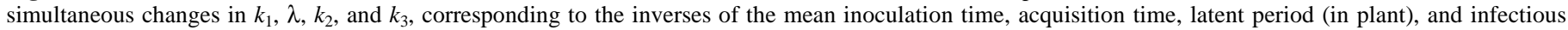

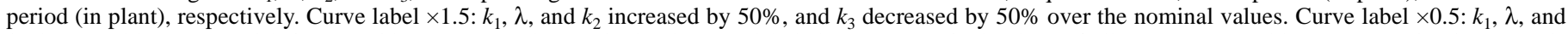

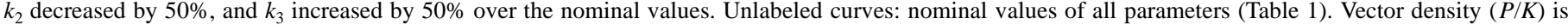

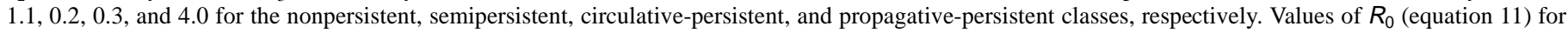

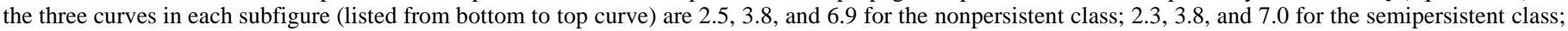
$0.97,3.8$, and 10.5 for the circulative-persistent class; and $0.70,3.8$, and 13.6 for the propagative-persistent class. 
the propagative transmission class, there was no apparent effect of different $V / P$ values (at $\psi=0$ ) on disease incidence (Fig. 13), partly because $1-\theta_{1}-\theta_{2}$ used here was about the same as the steadystate $Z / P$ value with no emigration $(Z * / P \approx 0.11$ [case 0 ]).

One further way that vector migration can be assessed is by plotting steady-state $d$ values $\left(d^{*}\right)$ (equation A3) versus $P / K$ for various combinations of $V / P$ and $\psi$ (and the nominal values of all other parameters). These relationships are shown in Figure 14 for four of the cases in Figure 13,0/1, 0, 0.25/1, and 0.25 (Table 3). Predictions from equation A3 were in agreement with the numerical solutions of equations 8 and 14 (or 15) at the values of $P / K$ used in Figure 13. When there was no introduction of infective insects into a field, either because $\psi=1$ or $\theta_{1}=1$ (the latter not considered here), all lost vectors were replaced by within-field repro-

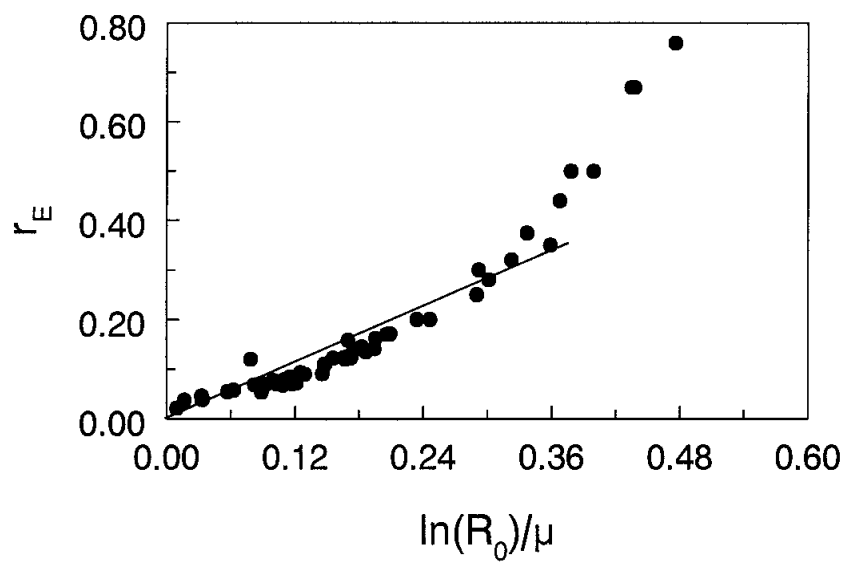

Fig. 11. Relationship between the basic reproductive number ( $R_{0}$; equation 11$)$, mean age of infectivity ( $\mu$; equation 17 ), and exponential rate of disease increase at small values of time $(t)$ for epidemics in Figures 5, 7, 8, 9, and 10 in which $R_{0}>1$. Straight line is the prediction from equation 16 . duction (or virus-free immigrants), and $d^{*}$ declined to 0 as $P / K$ decreased. The $P / K$ value at which $d^{*}$ was 0 corresponded to $R_{0}=$ $1 ; d^{*}$ was 0 at all lower $P / K$ values. For the situations with immigrating infective insects $\left(1-\theta_{1}-\theta_{2}>0\right), d^{*}$ never reached 0 as $P / K$ decreased (Fig. 14), because there were always some infective insects being introduced.

These $d^{*}$ values in Figure 14 confirm the results in Figure 13. That is, the propagative class was very sensitive to how lost insects were replaced ('births' versus immigrants [e.g., case 0.25 versus $0.25 / 1]$ ). Results similar to the propagative class can be seen for the other transmission classes only at low $P / K$ (with exact values dependent on class); at high $P / K$, however, $d^{*}$ values were similar for within-field reproduction and immigration situations, primarily because $d^{*}$ was close to 1 for all classes at high $P / K$. When there were no within-field reproduction (case 0 versus 0.25 ), there were only small differences in $d^{*}$ at most $P / K$ values, although the transitional values of $d$ could be farther apart (Fig. 13). The rank ordering of $d^{*}$ for cases 0 and 0.25 depended on the value of $Z * / P$ relative to $1-\theta_{1}-\theta_{2}$, as shown for the examples in Figure 13 .

\section{DISCUSSION}

Modeling of plant virus epidemics. The continuous-time, deterministic, and compartmental model of Jeger et al. (18) was successfully used here to evaluate theoretically the effects of transmission class on plant disease epidemics. The model can be written succinctly as a set of linked differential equations with parameters that have direct physical or biological meaning. With additionalalthough not required-assumptions about the (fixed) size of the insect population, it was possible to determine analytical results for steady-state values of disease incidence as a function of model parameters (Appendix). We expanded on previous work (18) by determining numerical solutions for a wide range of model parameters and showing the effects of parameters on both transitional and steady-state levels of disease for situations with and without
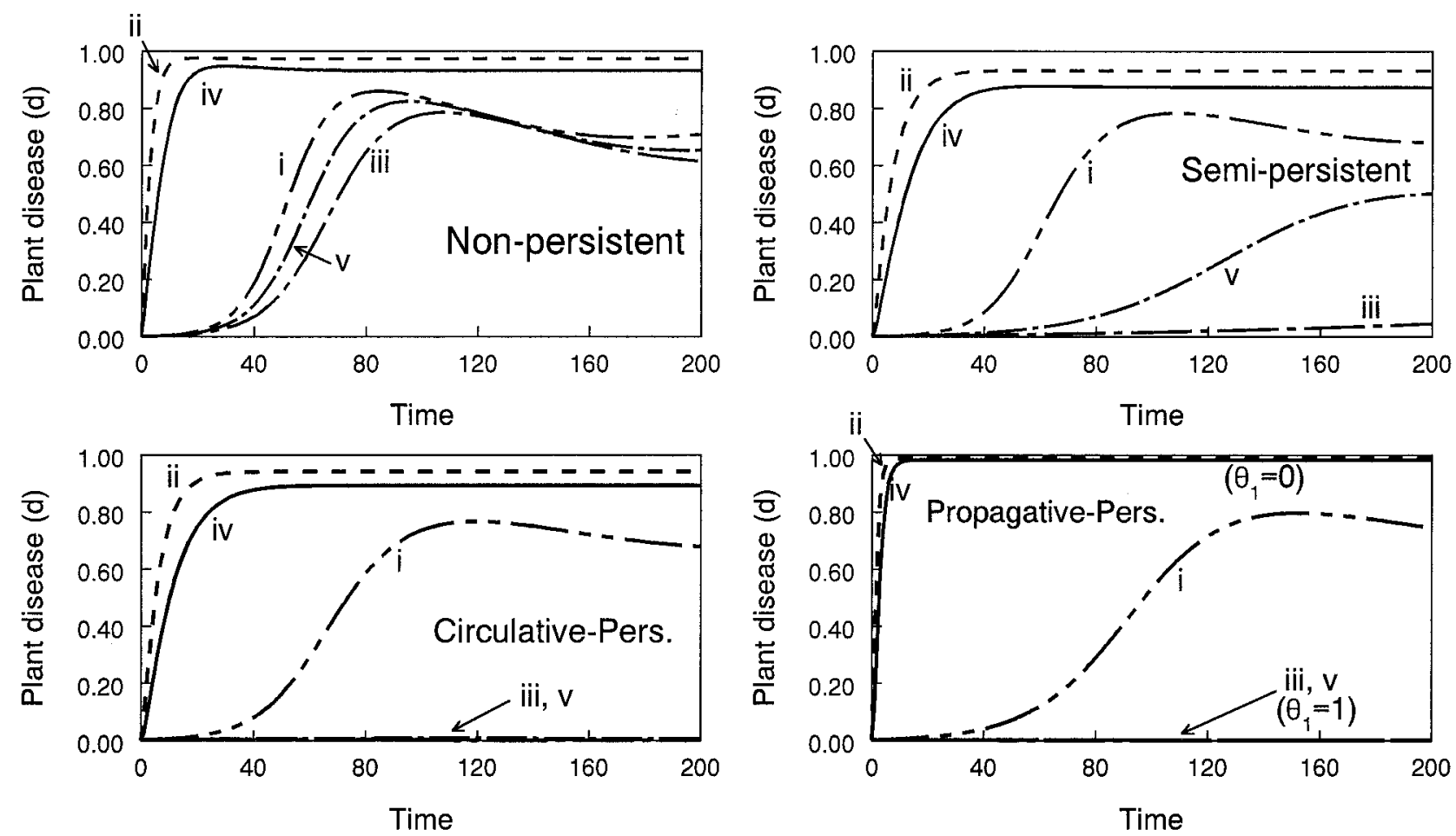

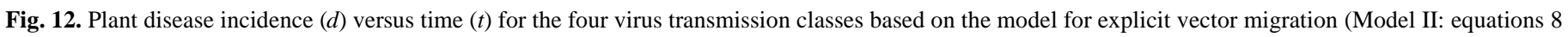

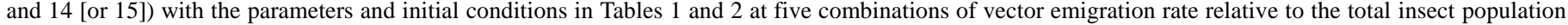

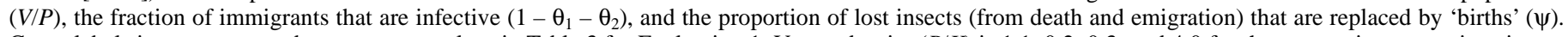

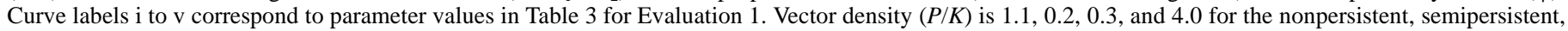
circulative-persistent, and propagative-persistent classes, respectively. $R_{0}$ (equation 11) is undefined for Model II when $\theta_{1}<1$. 
vector migration. Numerical solutions, with parameter values in Tables 1 and 2, yielded disease progress curves (Figs. 5 to 13) that are typical of many published epidemic data sets $(4,22,30,35-37)$.

Although other continuous-time compartmental models have been developed for virus diseases $(6,13,23,27)$, these either have not been formulated generally enough to encompass the full range of vectorvirus interactions characterizing nonpersistent, semipersistent, circulative-persistent, and propagative-persistent transmission classes (28) or all four plant infection categories $(H, L, S$, and $R$ ) (Fig. 2) and three vector-virus categories $(X, Y$, and $Z)$ were not considered. The objectives of these cited studies did not focus on the comparison of transmission classes, so such generalizations were unnecessary. Ferriss and Berger (8) did focus on a comparison of transmission classes in an innovative study by using a stochastic spatial-lattice discrete-time simulation model. Several other simulation and discrete-time models have been developed for specific virus diseases, with varying degree of detail, depending on the research objectives $(9,11,33)$. In general, these latter simulation models were used for very specific and detailed descriptions of a particular system. In the classification system of May (26), these would be labeled as tactical models. The model used in this paper, however, would be classified as strategic in the May framework, because we used it to "provide a conceptual framework for the discussion of broad classes of phenomena" and not to describe one particular plant pathosystem in detail (26). The Ferriss and Berger model $(2,8)$ could also be considered strategic, since it did not focus on a particular disease. However, their simulation approach does not allow for easy representation in equation format, making it more difficult to explore the general properties of the system of interest. For instance, although computer simulation makes it relatively easy to generate population growth curves in relation to many driving variables and parameters $(9,11,33)$, this approach rarely leads to the generic determination of conditions required for disease invasion (establishment) and persistence, as well as the steady-state and asymptotic values of disease (17). By basing the model in the current study on a relatively small number of (linked) equations, we have shown that steady-state values of disease incidence (and other variables) can be expressed in terms of model parameters (Appendix) (18) or the basic reproductive number $\left(R_{0}\right)$ (equation 11). Predictions of steady-state values of disease incidence, based on the equations derived by Jeger et al. (18), were found here to agree well with numerical solutions to the equations (equations 8 and 9 [or 10] for Model I and equations 8 and 14 [or 15] for Model II). Because of the complexity of the general model, however, transient behavior of the epidemics could generally only be assessed with numerical methods, although at small $t$, disease incidence could be approximated by an exponential equation for Model I (Fig. 11).

The nonpersistent viruses are different from the other viruses, especially the persistent ones, because of the very short times for acquisition and inoculation and the short latent and infectious periods in the vector (Table 1). In fact, the model developed here can be further simplified, as an approximation, if one assumes that both $\eta$ and $\tau$ are infinite, meaning that the latent and infectious periods are 0 . In the limit, one obtains the following equation for infective vectors if one holds $a \phi / \tau$ fixed $(=\sigma)$.

$$
z=\frac{\sigma\left(\frac{P}{K}\right) s}{1+\sigma s}
$$

One can substitute equation 18 for $z$ in equation 8 , thus eliminating all differential equations for the vector population. Based on this equation, the number of infective vectors per host plant is directly proportional to insect density $(P / K)$ and related to infectious plants $(s)$ in a saturation-type curve manner $(s /[1+\sigma s])$. Use of this approximation produces epidemic curves similar to those shown in the graphs of this paper (L. V. Madden, unpublished data). Modeling nonpersistent viruses in this way is analogous to the approaches taken by Madden et al. (23) and Marcus and Raccah (24) for this virus class.
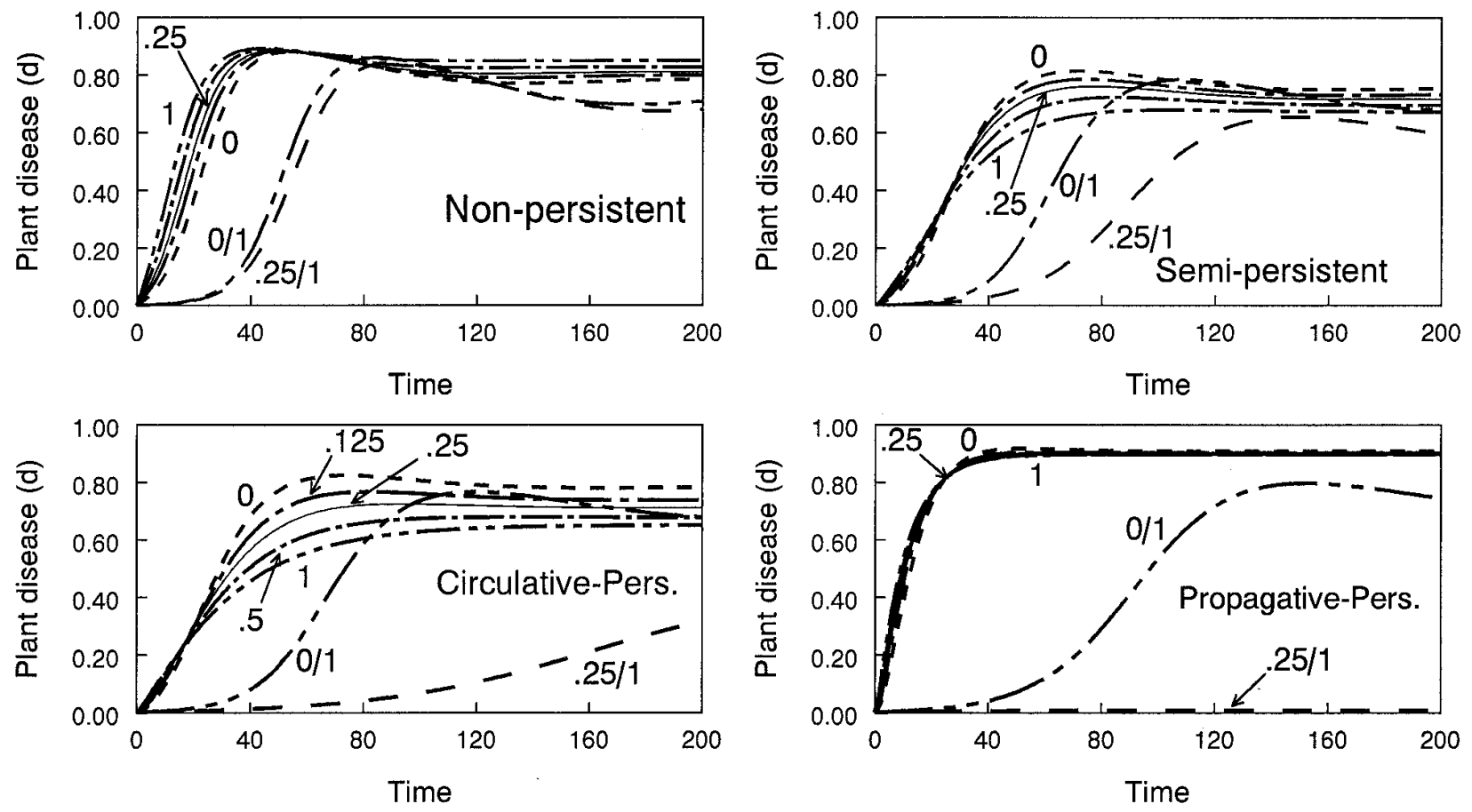

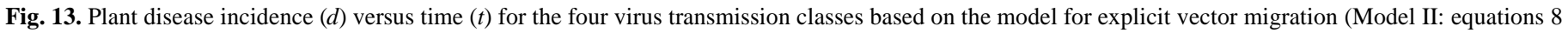

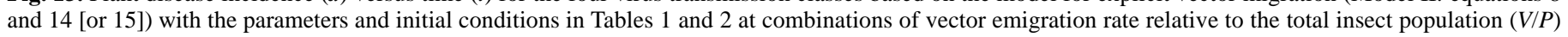

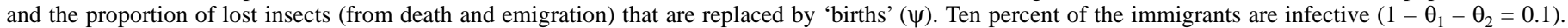

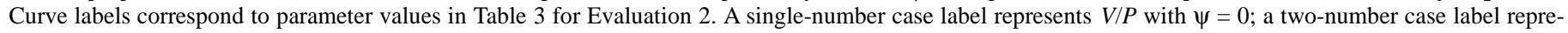

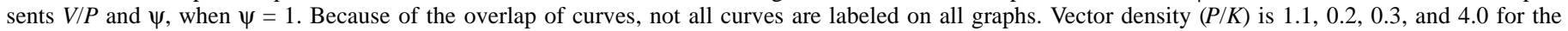
nonpersistent, semipersistent, circulative-persistent, and propagative-persistent classes, respectively. $R_{0}$ (equation 11 ) is undefined for Model II when $\theta_{1}<1$. 
Transmission classes and disease management. With the supposition that the parameter values in Table 1 were characteristic for the four transmission classes, it was found that these classes varied greatly in their epidemic behavior and in their sensitivity to changes in model parameters. For instance, two or three of the transmission classes could be easily identified based on epidemic response to vector density $(P / K)$ alone (Figs. 5 and 6). Although disease incidence increased with $P / K$ for all classes, very high values of $P / K$ were required for the propagative-persistent viruses to exhibit steady-state and transient values of $d$ similar to those found for the semipersistent and circulative-persistent viruses with very low $P / K$, all at nominal values of other parameters. The nonpersistent viruses were intermediate in terms of disease response to changes in $P / K$. However, small changes in vector activity $(\phi)$ led to very large changes in $d$ for the nonpersistent viruses, but large changes in $\phi$ had only a small effect on the propagative viruses. The other classes were intermediate in terms of response to $\phi$ (Fig. 8 ). For the propagative viruses, any increase in opportunity for inoculation of plants (by feeding on more plants per day) is negated by the decreasing probability of inoculation per plant visit. Conversely, changes in vector mortality $(\alpha)$-or mean lifetime $(1 / \alpha)$ — had no effect on nonpersistent viruses, only a little effect on semipersistent viruses, and a pronounced effect on the propagative viruses (Fig. 7). This is because, with the long times to acquire and inoculate (on average) coupled with the long latent period in the vector, increasing the time vectors are in a field greatly increases the probability of all of these events occurring in individual vectors for propagative viruses. With short times for all of these processes with nonpersistent and semipersistent viruses (Table 1; Figs. 1 and 3), the probability of all of these events occurring is high, even at large $\alpha$. It should be pointed out that small $\alpha$ (large $1 / \alpha$ ) here is analogous to the modeling situation of Ferriss and Berger (8), in which they found high $d$ with propagative viruses.

Virus disease management approaches that affect vectors directly (42), rather than the virus or vector-virus interaction, can be assessed in terms of the parameters $P / K, \alpha$, and $\phi$. Based on model results, reducing the number of vectors per plant (e.g., through the use of insecticides, host resistance to the insect, and cultural practices) will have a large effect on propagative viruses and, to a somewhat lesser extent, nonpersistent viruses (Fig. 5). However, the reduction in vector density will have to be substantial to have any noticeable effect on semipersistent and circulative viruses, which may explain the difficulty in controlling epidemics of the geminivirus African cassava mosaic virus in Africa (13). Moreover, reducing vector density will not be effective for nonpersistent viruses if insect mobility (plants per day; $\phi$ ) is high (Fig. 8). It is known that many insecticides can actually increase mobility of insects (at least temporarily), as individuals move from plant to plant to escape the insecticide (or find a suitable host) $(31,34)$. Such an increase in mobility will not interfere with the control of the propagative viruses (Fig. 8) because of reduced time per plant visit for a vector to transmit, but will be an obstacle for controlling nonpersistent viruses. For the persistent viruses, especially the propagative ones, reducing the lifetime of vectors $(1 / \alpha)$ will be a very efficient means of control, at any vector density (Fig. 7). On the other hand, reducing the lifetime of vectors will have no effect on nonpersistent viruses unless vector density is low-in particular, killing the current vectors in a field will not be effective if new vectors are entering the field.

Increasing the resistance of the plant to the virus $(21,29,37)$ can be quantified through the parameters for the rate at which the virus moves through the latent $\left(k_{2}\right)$ and infectious periods $\left(k_{3}\right)(4,6$, $15)$, as well as for the acquisition $(\lambda)$ and inoculation $\left(k_{1}\right)$ rates. Of course, increasing resistance to plant feeding by the vector (independent of the virus) could also affect $\lambda$ and $k_{1}$ (42). Changing $k_{2}$ had no long-term effect on viruses in any of the transmission classes, as quantified by steady-state (equations 12 and A3) and asymptotic values of disease incidence (equation 13) (Fig. 9). This was expected based on the minor role that $k_{2}$ has in determining
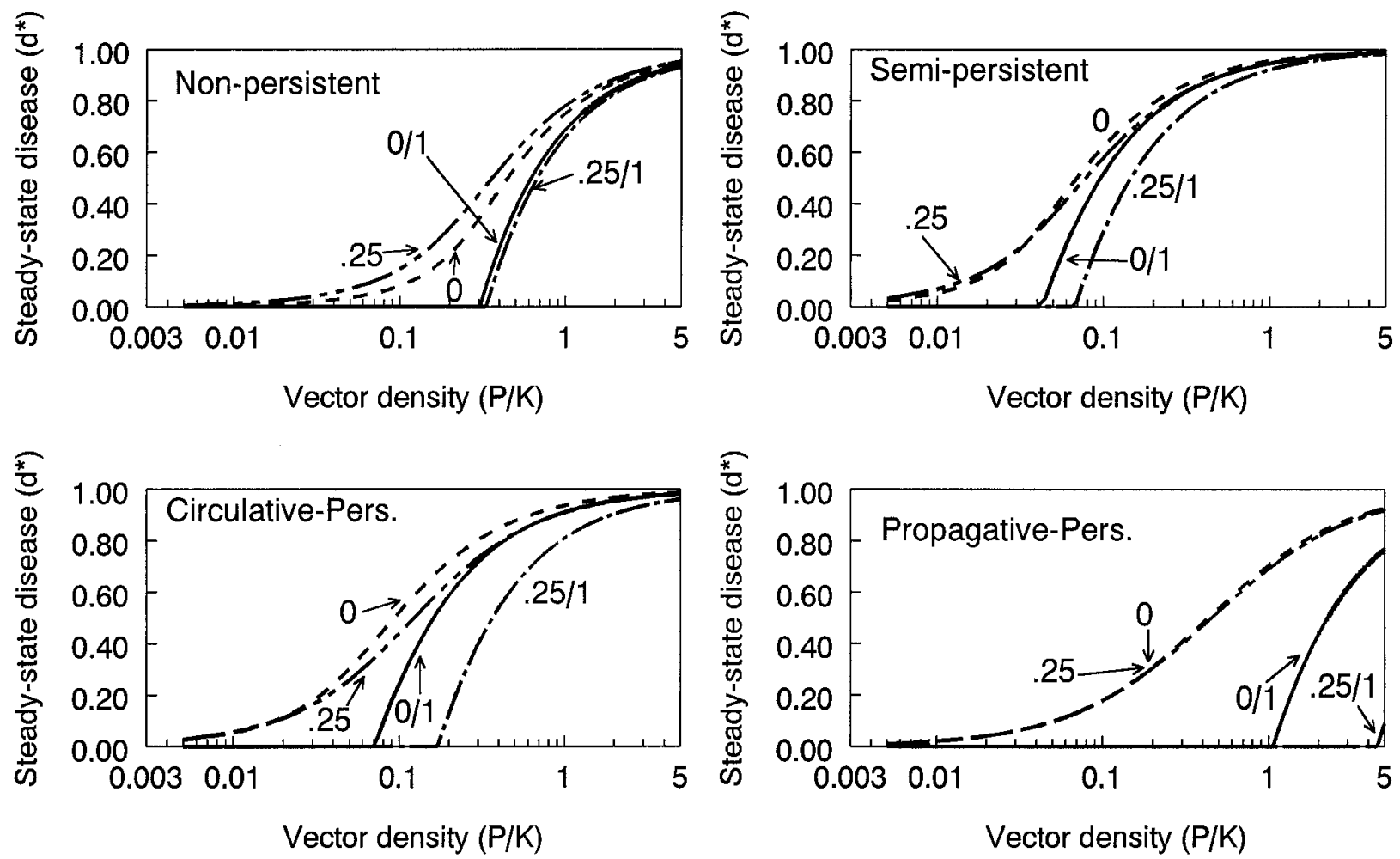

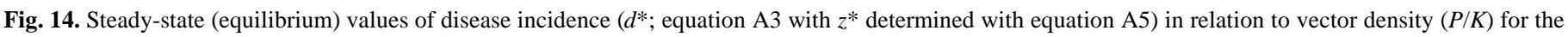

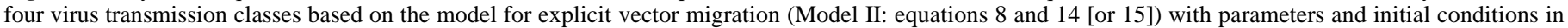

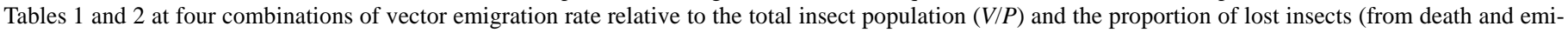

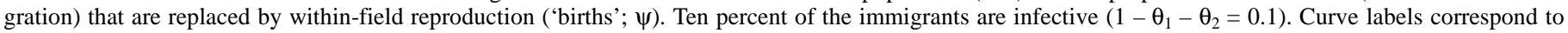
parameter values in Table 3 for Evaluation 2. 
$R_{0}$ (equation 11); in fact, many theoretical epidemiology studies ignore the latent category to simplify the mathematics and focus on steady-state values $(1,10)$. However, $k_{2}$ did have a moderate effect on transitional disease dynamics at small and moderate $t$, especially for those virus transmission classes with a short latent and infectious period in the vector (e.g., nonpersistent viruses) (Fig. 9A); that is, decreasing $k_{2}$ (increasing the latent period in the plant), which can be accomplished through resistance, reduced disease development early in the epidemic. This is consistent with the epidemiological result that $r_{E}$ is inversely proportional to the latent period (a component of $\mu$ [equation 17]) and directly proportional to $\ln \left(R_{0}\right)$ (equation 16) (5). In contrast to $k_{2}$ results, decreasing the time that plants are infectious (increasing $k_{3}$ ) had a fairly large effect on reducing transitional and steady-state disease values for all four transmission classes (Fig. 9).

Changing $k_{1}$ and $\lambda$ had a differential effect on the four transmission classes. In particular, reducing these two rates by $50 \%$ had virtually no effect on the nonpersistent and semipersistent viruses (Fig. 9). This is because, with the high nominal rates for these two classes (Table 1), the probabilities of acquisition and inoculation per plant visit ( $a$ and $b$ [equations 3 and 5]) were about 1, even with a large reduction in $k_{1}$ and $\lambda$. Conversely, changing these rates for the two persistent classes had a direct effect on disease development (Figs. 9D and 10), because $a$ and $b$ are close to linearly related to $\lambda$ and $k_{1}$ at these lower values. Thus, effective control of nonpersistent viruses through resistance to acquisition or inoculation will require a much greater percentage of reduction in the acquisition/inoculation rates relative to the persistent classes.

Large differences could be seen among the four transmission classes (Table 1) in terms of epidemic responses to the migration parameters of emigration rate $(V$ or $V / P)$, fraction of lost vectors (from emigration and death) that are replaced by immigrants $(1-\psi)$, and fraction of the immigrants that are in the infective $\left(1-\theta_{1}-\theta_{2}\right)$ state (Model II). Results depended heavily on whether or not lost insects were replaced solely by 'births' (within-field reproduction; $\psi=1$ ) or some immigrants $(\psi<1)$ and whether viruliferous insects were in the immigrant pool $\left(\theta_{1}<1\right.$, so that $\left.1-\theta_{1}-\theta_{2}>0\right)$ or not $\left(\theta_{1}=1\right)$. For all transmission classes, disease development was slower when lost insects were replaced exclusively by 'births' $(\psi=1)$ than by infective immigrants $(\psi=0)$ (Figs. 12 and 13). The propagative viruses were very sensitive and the nonpersistent viruses were only slightly sensitive to 'births' versus immigration (in the situation with $V / P>0$ ). Because of the short times to acquire and inoculate, the nonpersistent viruses were little affected by the source of vectors (inside or outside the field). When immigration represented a substantial fraction of the new insects in a field $(\psi \leq 0.5)$, epidemic results varied with $1-\theta_{1}-\theta_{2}$. For instance, disease development was reduced, possibly to 0 , when all immigrants were virus free $\left(1-\theta_{1}-\theta_{2}=0\right)$ and increased to high levels when immigrants were infective $\left(1-\theta_{1}-\theta_{2}=1\right)$ (Fig. 12). The two persistent classes were most sensitive to this, primarily because of the long times needed for a new vector in a field to acquire the virus and then inoculate a susceptible plant. Somewhat surprisingly, change in VIP had only a small effect on the epidemics for any transmission class when immigrants (with $10 \%$ being infective) replaced lost vectors (Fig. 13, cases 0 to 1 ). Results for different values of $V / P$ depended more on the value of 1 $\theta_{1}-\theta_{2}$ relative to the fraction of infective insects in the total vector population $(Z / P)$ than on transmission class.

Because results were much more sensitive to $\psi$ and $1-\theta_{1}-\theta_{2}$ than to $V / P$, the former two can be considered in more detail in terms of disease management. Obviously, when there are no immigrants $(\psi=1)$, management can focus on the other factors considered with Model I. In situations in which immigrants are an important source of vectors in crop plants $(\psi<1)$, it is advantageous to reduce $1-\theta_{1}-\theta_{2}$ to as close to 0 as possible for all transmission classes, but especially for the circulative viruses at any vector density and the propagative viruses at high vector density (Fig. 12, cases iii and v). Values of $1-\theta_{1}-\theta_{2}$ as high as 0.10 (Fig. 13) are still too large for effective control under the nominal conditions here. In fact, any value of $1-\theta_{1}-\theta_{2}>0$ will lead to disease persistence (Fig. 14), but depending on the other conditions (parameters), reducing $1-\theta_{1}-\theta_{2}$ to close to $0-$ possibly by eliminating infected plants in weeds and surrounding crops-should be effective for maintaining disease at a low level. With nonpersistent viruses, however, such a reduction will be far less effective if insect density in the field of interest is not low and no control of the disease is done within the field, since insects can acquire and then transmit viruses so quickly.

Synopsis. The theoretical research presented here elucidating the effects of transmission class on plant disease epidemics can be summarized through a consideration of a model epidemic. When there is no vector migration or when the population of insects is the same globally as locally (Model I), $d$ increases exponentially early in the transitional phase of an epidemic (when $d \lesssim 0.05$ ) if the net reproductive number $\left(R_{0}\right)$ (equation 11 ) is greater than 1 . As shown in Figure 11, the relative rate of increase in $d$ at these early times, $r_{E}$, is directly related to $R_{0}$ and the mean age when an individual insect or plant is infectious, $\mu$ (equation 17). The relationship, although not exact, is well described by equation 16 at low $R_{0}$. Both $\mu$ and $R_{0}$ are directly related to easily interpreted parameters that characterize the transmission process, behavior of the virus in the plant and insect, or vector population dynamics. Thus, by using the simple exponential model $d=d_{0} \cdot \exp \left(r_{E} t\right)$ and the relationship between $r_{E}, \mu$, and $R_{0}$, one can approximately assess the effects of the transmission process and disease control strategies on epidemics without the use of complicated nonlinear differential-equation models (equations 8 and 9 [or 10]).

At (much) longer time periods, $d$ eventually reaches a steady state $\left(d^{*}\right)$, defined by equation 12 (or equations $\mathrm{A} 1$ and $\mathrm{A} 3$ ), or approaches an asymptotic upper limit $\left(d_{\infty}\right)$, roughly approximated by equation 13 . Both of these terms are directly related to $R_{0}$. The expression $1-\left(R_{0}\right)^{-1}$ provides an especially useful term to characterize long-term disease dynamics without the use of differential equations, because it is the upper limit for $d^{*}$ (equation 12) when plant host mortality and regrowth $(\beta)$ is nonzero but low. When $\beta=$ $0 /$ day, a steady state cannot be achieved, but a final disease outcome is achieved that we have found numerically to be in the interval, $1-\left(R_{0}\right)^{-1}<d_{\infty}<1$ (equation 13 ); $d_{\infty}$ quickly approaches 1 as $R_{0}$ increases above 4 .

Between the early exponential and later steady-state (or asymptotic) stages, there is a period in which predictions of $d$ are only possible through numerical solutions of the differential equations (equations 8 and 9 [or 10]). Although, in some cases, especially when plant host and regrowth is $0, d$ increases in roughly a logistic manner during part of this period, in most cases with $\beta$ > 0/day, $d$ somewhat overshoots its steady-state value during this transitional stage (Fig. 6A), before eventually stabilizing at $d^{*}$.

The situation is more complicated when the insect immigrants are not in the same categories as the emigrants, such that explicit terms for migration are needed (Model II). In general, the increase in $d$ early in the epidemic is a mixture of exponential increase from the insects internal to the field and linear increase from immigrating viruliferous insects (when $d \lesssim 0.05$ ). However, when the immigrants are all virus free $\left(\theta_{1}=1\right), R_{0}$ can still be defined and the increase will be exponential. After the transitional stage of disease development, represented by numerical solutions to equations 8 and 14 (or 15 ), $d$ reaches a steady-state value $\left(d^{*}\right)$ when $\beta$ > $0 /$ day, which is determined based on the same parameters that pre$\operatorname{dict} d^{*}$ for Model I, plus the terms for insect emigration rate $(V)$, fraction of lost insects replaced by immigrants $(1-\psi)$, and virus status of the immigrants $\left(1-\theta_{1}-\theta_{2}\right)$. The $d^{*}$ values, determined from equations $\mathrm{A} 5$ and $\mathrm{A} 3$, are lower than 1 , and can be higher or lower than those found with Model I (Figs. 12 to 14). Because of the complicated nature of equations A5 and A3, little insight is 
obtainable into epidemic behavior in relation to transmission or other characteristics for Model II without use of numerical results. Numerical solutions for Model II epidemics, as well as for the transitional phase of Model I epidemics, do considerably extend the understanding we can have of the plant virus disease epidemic process.

\section{APPENDIX}

Model I. Based on equations 8 and 9, steady-state values of the plant and insect population values can be derived (18). The steadystate value of infective vectors $(z)$, conditioned on $\beta>0 /$ day, can be written as

$$
z^{*}=\frac{\beta\left\{a b \phi^{2}\left[\frac{P}{K}\right] k_{2}-[\alpha+\eta]\left[\frac{\tau+\alpha(1-q)}{\eta}\right]\left[k_{2}+\beta\right]\left[k_{3}+\beta\right]\right\}}{b \phi\left\{[\alpha+\eta]\left[\frac{\tau+\alpha(1-q)}{\eta}\right]\left[k_{2}+\beta\right]\left[k_{3}+\beta\right]+a \phi k_{2} \beta\left[\frac{\tau+\alpha(1-q)}{\eta}+1\right]\right\}}
$$

in which the asterisk indicates the equilibrium value, and all other terms are defined in Table 2. Equation A1 can also be written more succinctly as

$$
z^{*}=\frac{\beta\left(R_{0}-1\right)}{b \phi\left(1+\frac{C_{1}}{C_{2}}\right)}
$$

in which

$$
C_{1}=a \phi k_{2} \beta\left[\frac{\tau+\alpha(1-q)}{\eta}+1\right]
$$

and

$$
C_{2}=[\alpha+\eta]\left[\frac{\tau+\alpha(1-q)}{\eta}\right]\left[k_{2}+\beta\right]\left[k_{3}+\beta\right]
$$

and $R_{0}$ is given by equation 11 . Equation A2 clearly shows the effect of $R_{0}$ on steady-state values, in that $z^{*}$ is only greater than 0 if $R_{0}>1$. The steady-state value of $d\left(d^{*}\right)$ is given by

$$
d^{*}=1-\frac{\beta}{\beta+b \phi z^{*}}
$$

in which $z^{*}$ is determined from equation A1 when $\beta>0 /$ day. Substitution of equation A1 for $z^{*}$ in equation A3 and algebraic manipulation results in the following simple expression for $d^{*}$.

$$
d^{*}=\frac{R_{0}-1}{R_{0}+\frac{C_{1}}{C_{2}}}
$$

At very small $\beta, C_{1} / C_{2} \approx 0$, resulting in $1-\left(R_{0}\right)^{-1}$ as an approximation for $d^{*}$ (equation 12). Values of $l^{*}, s^{*}$, and $y^{*}$ can be determined from the equations by Jeger et al. (18), with $a$ substituted for $\lambda T$, and $b$ for $k_{1} T$.

Model II. Equations for steady-state values of insect population variables are much more complicated when there is explicit vector immigration and emigration (equations 8 and 14 [or 15]). The steady-state value of $z$ when $\beta>0 /$ day is one of two roots of a quadratic equation, specifically

$$
z^{*}=\frac{-C_{3}-\sqrt{\left(C_{3}^{2}+4 C_{4} C_{5}\right)}}{-2 C_{4}}
$$

in which $C_{3}$ to $C_{5}$ are functions of the model parameters. These functions are

$$
\begin{aligned}
& C_{3}=A_{4} \phi b+A_{1} a b \phi^{2}\left(\frac{P}{K}+A_{3}\right)-\beta A_{2} A_{5}+A_{2} A_{5} \phi b \\
& C_{4}=A_{1} \phi^{2} a b\left(A_{2}+1\right)+A_{2} A_{5} \phi b \\
& C_{5}=A_{4} \beta+\beta A_{3} A_{5}
\end{aligned}
$$

with the coefficients $A_{1}$ to $A_{5}$ given by

$$
\begin{aligned}
& A_{1}=\left(\frac{k_{2}}{k_{2}+\beta}\right)\left(\frac{\beta}{k_{3}+\beta}\right) \\
& A_{2}=\frac{\tau+\left(\alpha+\frac{V}{P}\right)(1-q \psi)}{\eta} \\
& A_{3}=\frac{(1-\psi)\left(1-\theta_{1}-\theta_{2}\right)(V+\alpha P)}{\eta K} \\
& A_{4}=\frac{(1-\psi) \theta_{2}(V+\alpha P)}{K} \\
& A_{5}=\alpha+\eta+\frac{V}{P}
\end{aligned}
$$

Steady-state disease incidence $\left(d^{*}\right)$ is given by equation $\mathrm{A} 3$, but with equation A5 substituted for $z^{*}$. Other equations are given by Jeger et al. (18).

\section{ACKNOWLEDGMENTS}

Salaries and research support for L. V. Madden were provided by state and federal funds appropriated to the Ohio Agricultural Research and Development Center, The Ohio State University. IACR-Rothamsted receives grant-aided support from the Biotechnology and Biological Sciences Research Council of the United Kingdom.

\section{LITERATURE CITED}

1. Anderson, R. M., and May, R. M. 1991. Infectious Diseases of Humans: Dynamics and Control. Oxford University Press, Oxford, United Kingdom.

2. Berger, P. H., and Ferriss, R. S. 1989. Mechanisms of arthropod transmission of plant viruses: Implication for the spread of disease. Pages 4084 in: Spatial Components of Plant Disease Epidemics. M. J. Jeger, ed. Prentice-Hall, Englewood, NJ.

3. Berger, P. H., Zeyen, R. J., and Groth, J. V. 1987. Aphid retention of maize dwarf mosaic virus: Epidemiological implications. Ann. Appl. Biol. 111:337-344.

4. Campbell, C. L., and Madden, L. V. 1990. Introduction to Plant Disease Epidemiology. Wiley Interscience, New York.

5. Caswell, H. 1989. Matrix Population Models: Construction, Analysis and Interpretation. Sinauer Publishing, Sunderland, MA.

6. Chan, M. S., and Jeger, M. J. 1994. An analytical model of plant virus disease dynamics with roguing and replanting. J. Appl. Ecol. 31:413-427.

7. Diekmann, O., Heesterbeek, J. A. P., and Metz, J. A. J. 1990. On the definition and computation of the basic reproduction ratio $R_{0}$ in models for infectious-diseases in heterogeneous populations. J. Math. Biol. 28:365-382.

8. Ferriss, R. S., and Berger, P. H. 1993. A stochastic simulation model of epidemics of arthropod-vectored plant diseases. Phytopathology 83:1269-1278.

9. Frazer, B. D. 1977. Plant virus epidemiology and computer simulation of aphid populations. Pages 413-431 in: Aphids and Virus Vectors. K. R. Harris and K. Maramorosch, eds. Academic Press, New York.

10. Gilligan, C. A., Gubbins, S., and Simons, S. A. 1997. Analysis and fitting of an SIR model with host response to infection load for a plant disease. Philos. Trans. R. Soc. Lond. B 352:353-364.

11. Gutierrez, A. P., Havenstein, D. E., Nix, H. A., and Moore, P. A. 1974. The ecology of Aphis craccivora Koch and subterranean clover stunt virus in south-east Australia. J. Appl. Ecol. 11:1-20.

12. Holt, J., and Chancellor, T. C. B. 1996. Simulation modelling of the spread of rice tungro virus disease: The potential for management by roguing. J. Appl. Ecol. 33:927-936.

13. Holt, J., Jeger, M. J., Thresh, J. M., and Otim-Nape, G. W. 1997. An epidemiological model incorporating vector population dynamics applied to African cassava mosaic virus disease. J. Appl. Ecol. 34:793-806.

14. Irwin, M. E., and Ruesink, W. G. 1986. Vector intensity: A product of propensity and activity. Pages 13-33 in: Plant Virus Epidemics: Monitoring, Modelling and Predicting Outbreaks. G. D. McLean, R. G. Garrett, and W. G. Ruesink, eds. Academic Press, Sydney, Australia.

15. Jeger, M. J. 1982. The relation between total, infectious, and postinfectious diseased plant tissue. Phytopathology 72:1185-1189.

16. Jeger, M. J., and van den Bosch, F. 1994. Threshold criteria for model plant disease epidemics. I. Asymptotic results. Phytopathology 84:24-27. 
17. Jeger, M. J., and van den Bosch, F. 1994. Threshold criteria for model plant disease epidemics. II. Persistence and endemicity. Phytopathology 84:28-30.

18. Jeger, M. J., van den Bosch, F., Madden, L. V., and Holt, J. 1998. A model for analysing plant-virus transmission characteristics and epidemic development. IMA (Inst. Math. Appl.) J. Math. Appl. Med. Biol. 15:1-18.

19. Keeling, M. J. 1999. The effects of local spatial structure on epidemiological invasions. Proc. R. Soc. Lond. B 266:859-867.

20. Kennedy, J. S., Day, M. F., and Eastop, V. F. 1962. A Conspectus of Aphids as Vectors of Plant Viruses. Commonwealth Institute of Entomology, London.

21. Khetarpal, R. K., Maisonneuve, B., Maury, Y., Chalhoub, B., Dinant, S., Lecoq, H., and Varma, A. 1998. Breeding for resistance to plant viruses. Pages 14-32 in: Plant Virus Disease Control. A. Hadidi, R. K. Khetarpal, and H. Koganezawa, eds. The American Phytopathological Society, St. Paul, MN.

22. Madden, L. V., Pirone, T. P., and Raccah, B. 1987. Temporal analysis of two viruses increasing in the same tobacco fields. Phytopathology 77: 974-980.

23. Madden, L. V., Raccah, B., and Pirone, T. P. 1990. Modelling plant disease increase as a function of vector numbers: Nonpersistent viruses. Res. Popul. Ecol. 32:47-65.

24. Marcus, R., and Raccah, B. 1986. A model for spread of non-persistent virus diseases. J. Appl. Stat. 13:167-175.

25. Matthews, R. E. F. 1991. Plant Virology. 3rd ed. Academic Press, New York.

26. May, R. M. 1974. Stability and Complexity in Model Ecosystems. 2nd ed. Princeton University Press, Princeton, NJ.

27. Nakasuji, F., Miyai, S., Kawamoto, H., and Kiritani, K. 1985. Mathematical epidemiology of rice dwarf virus transmitted by green rice leafhoppers: A differential equation model. J. Appl. Ecol. 22:839-847.

28. Nault, L. R. 1997. Arthropod transmission of plant viruses: A new synthesis. Ann. Entomol. Soc. Am. 90:521-541.

29. Nutter, F. W. 1993. Quantification of components contributing to ratereducing resistance in a plant virus pathosystem. Pages 297-308 in: Systems Approaches for Agricultural Development. F. W. T. Penning de Vries, ed. Kluwer Publishing, Dordrecht, the Netherlands.

30. Nutter, F. W. 1997. Quantifying the temporal dynamics of plant virus epidemics: A review. Crop Prot. 16:603-618.
31. Perring, T. M., Gruenhagaen, N. M., and Farrar, C. A. 1999. Management of plant viral diseases through chemical control of insect vectors. Annu. Rev. Entomol. 44:457-481.

32. Ross, R. 1911. The Prevention of Malaria. 2nd ed. Murray Publishing, London.

33. Ruesink, W. G., and Irwin, M. E. 1986. Soybean mosaic virus epidemiology: A model and some implications. Pages 295-313 in: Plant Virus Epidemics: Monitoring, Modelling and Predicting Outbreaks. G. D. McLean, R. G. Garrett, and W. G. Ruesink, eds. Academic Press, Sydney, Australia.

34. Satapathy, M. K. 1998. Chemical control of insect and nematode vectors of plant viruses. Pages 188-195 in: Plant Virus Disease Control. A. Hadidi, R. K. Khetarpal, and H. Koganezawa, eds. The American Phytopathological Society, St. Paul, MN.

35. Thresh, M. J. 1978. The epidemiology of plant virus diseases. Pages 7991 in: Plant Disease Epidemiology. P. R. Scott and A. Bainbridge, eds. Blackwell, Oxford.

36. Thresh, M. J. 1980. An ecological approach to the epidemiology of plant virus diseases. Pages 57-70 in: Comparative Epidemiology; A Tool for Better Disease Management. J. Palti and J. Kranz, eds. PUDOC Publishing, Wageningen, the Netherlands.

37. Thresh, M. J. 1983. Progress curves of plant virus disease. Appl. Biol. 8: $1-85$.

38. Vanderplank, J. E. 1975. Principles of Plant Infection. Academic Press, New York.

39. Waterworth, H. E., and Hadidi, A. 1998. Economic losses due to plant viruses. Pages 1-13 in: Plant Virus Disease Control. A. Hadidi, R. K. Khetarpal, and H. Koganezawa, eds. The American Phytopathological Society, St. Paul, MN.

40. Watson, M. A. 1960. The ways in which plant viruses are transmitted by vectors. Pages 157-161 in: Rep. 7th Commonw. Entomol. Conf. Eastern Press, London.

41. Watson, M. A., and Roberts, F. M. 1939. A comparative study of the transmission of Hycocyamus virus 3, potato virus $\mathrm{Y}$ and cucumber virus 1 by vectors Myzus persicae (Sulz), M. circumflexus (Buckton), and Macrosiphum gu (Koch). Proc. R. Soc. Lond. B 127:543-576.

42. Zitter, T. A., and Simons, J. N. 1980. Management of viruses by alteration of vector efficiency and by cultural practices. Annu. Rev. Phytopathol. 18:289-310. 